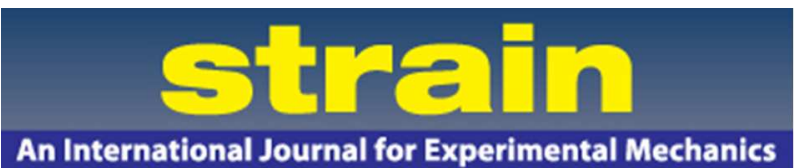

\title{
Smoothly Varying In-Plane Stiffness Heterogeneity Evaluated Under Uniaxial Tensile Stress
}

\begin{tabular}{|r|l|}
\hline Journal: & Strain \\
\hline Manuscript ID & STRAIN-1279.R1 \\
\hline Manuscript Type: & Special Issue Article \\
\hline Date Submitted by the Author: & 22-May-2017 \\
\hline Complete List of Authors: & $\begin{array}{l}\text { Considine, John; USDA, FS, Forest Products Laboratory, Fiber \& Chemical } \\
\text { Sciences } \\
\text { Pierron, Fabrice; University of Southampton, Faculty of Engineering and } \\
\text { the Environment } \\
\text { Turner, Keven; University of Pennsylvania, Mechanical Engineering and } \\
\text { Applied Mechanics } \\
\text { Lava, Pascal; MatchID Metrology Beyond Colors } \\
\text { Tang, Xiaoyan; USDA, FS, Forest Products Laboratory, Fiber \& Chemical } \\
\text { Sciences }\end{array}$ \\
\hline Keywords: & VFM, stiffness, heterogeneity, DIC, uniaxial tension \\
\hline \multicolumn{2}{|l}{} \\
\hline
\end{tabular}




\title{
Smoothly Varying In-Plane Stiffness Heterogeneity Evaluated Under Uniaxial Tensile Stress
}

\author{
J.M. Considine \\ USDA Forest Service, Forest Products Laboratory, USA \\ 608.231 .9525 \\ F. Pierron \\ Engineering and the Environment, University of Southampton, UK
}

\author{
K.T. Turner \\ Mechanical Engineering and Applied Mechanics, University of Pennsylvania, USA \\ P. Lava \\ MatchID n.v., Belgium \\ X. Tang \\ USDA Forest Service, Forest Products Laboratory, USA
}

\begin{abstract}
Identification of spatially varying stiffness is a challenging, but important, research topic in the mechanics of materials and can provide the necessary information for material suitability, damage and process control, especially for high-value applications. One homogeneous and three heterogeneous virtual field method (VFM) formulations were used to create a methodology for varying stiffness identification. Two FEM simulations were used to demonstrate the validity and capability of the formulations. A tensile test protocol was implemented to identify spatially varying stiffness of uniaxial tensile paperboard specimens using digital image correlation (DIC). A recently published form of VFM, which enforces global equilibrium in the spatial frequency domain, was used to better examine smoothly varying stiffnesses. Two tensile specimens of a single commercial paperboard, known to contain heterogeneities, were examined, and local stiffnesses were identified. Examination of local equilibrium with VFM was used to explain differences in stiffness identification. A polyethylene terephthalate (PET) specimen was used to demonstrate homogeneity.
\end{abstract}

Keywords: VFM, stiffness, heterogeneity, DIC, uniaxial tension 


\section{Nomenclature}

$\partial S \quad$ Portion of $S$ on which $\bar{T}_{i}$ acts

$\varepsilon_{i} \quad$ Strain in object; $i=x, y$ or $s$

$\varepsilon_{e q} \quad$ Von Mises equivalent strain

$\varepsilon_{i}^{*} \quad$ Virtual strains in object: $i=x, y, s$

$\checkmark \quad$ In-plane Poisson ratio

$v^{(j)} \quad$ In-plane Poisson ratio for region $S^{(j)}$

$\sigma_{i} \quad$ Stresses in object; $i=x, y, s$

$\sigma_{i}^{*} \quad$ Virtual stresses in object; $i=x, y, s$

$a_{m, n} ; b_{m, n} \quad$ Fourier coefficients

$L_{x} ; L_{y} \quad x, y$ dimensions of $S$

$l \quad$ Boundary of $S$

$m ; n \quad$ Fourier spatial frequency components

$N \quad$ Maximum value of $m, n$

$Q \quad$ Isotropic, in-plane stiffness; $Q=E / 1-v^{2}$ and $E$ is Young's modulus

$Q(x, y) \quad$ In-plane stiffness from Fourier analysis

$Q^{(j)} \quad$ Isotropic, in-plane stiffness for region $S^{(j)}$

$Q^{\text {Reuss }} \quad$ Reuss stiffness (inverse of average compliance)

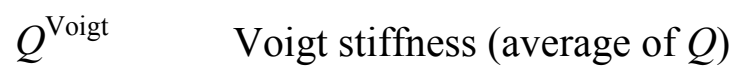

$\rho \quad$ Density, $\mathrm{kg} \cdot \mathrm{m}^{-3}$

S-D surface of object being examined

$S^{(j)} \quad$ Area portion of $S$ such that $S=\sum_{j=1}^{3} S^{(j)}$; three subdivisions of $S$ used here

$S^{\prime} \quad$ Area portion of $S$ where equilibrium is examined for equilibrium gap procedure

$s \quad$ In-plane shear direction

$\bar{T}_{i} \quad$ Surface traction on $S$ in direction $i$ 
1

2

3

4

5

6

7

8

9

10

11

12

13

14

15

16

17

18

19

20

21

22

23

24

25

26

27

28

29

30

31

32

33

34

35

36

37

38

39

40

41

42

43

44

45

46

47

48

49

50

51

52

53

54

55

56

57

58

59

60 $u_{i}^{*} \quad$ Virtual displacements; $i=x, y$ or $s$

$V \quad$ Ultrasonic velocity, $\mathrm{km} \cdot \mathrm{s}^{-1}$

$x ; y \quad$ Axes for Cartesian coordinate system 


\section{Introduction}

Characterization of the behavior of heterogeneous materials has broad applications in mechanics. For some materials, such as fiber/epoxy laminates, heterogeneity is intentionally introduced via stiff fibers to increase stiffness and strength. Conversely, some materials, such as a cellulose fiber composite like paperboard, have unintentional heterogeneities resulting from manufacturing. Other materials are initially homogeneous but become heterogeneous during use, as in the case of impact damage. Characterization of these and other heterogeneous materials is important for their continued use and extension to new product applications. The objective of this work was to create a methodology to identify the location and magnitude of smoothly varying stiffness heterogeneities.

Recent advancements in computer vision and analysis systems, such as digital image correlation (DIC), can provide data with sufficient resolution that inhomogeneities can be observed and evaluated. Evaluation techniques have also advanced and are now able to incorporate this full-field high-precision data in an analytically rigorous manner. Coupling of experimental techniques with material evaluation can provide the means to confidently characterize heterogeneous material behavior.

One of these material evaluations is the virtual fields method (VFM) [1], which uses a global equilibrium methodology and is most often used to identify stiffness parameters. VFM has been used in different formulations to identify heterogeneous stiffness. Using magnetic resonance imaging to locate and determine geometry of a heterogeneity, Avril et al. [2] used VFM to determine the ratio of stiffness between a stiff inclusion and a soft matrix. Bucaro et al. [3] used manual virtual fields to examine a finite element method (FEM) simulation of inclusions in plates while in bending. Using the same approach, but on experimental data, Romano et al. [4] used laser Doppler vibrometry to examine rib detachment in reinforced plates. The group then extended their work with a simulation to inspect a wood beam for defects [5]. Several researchers [6 - 8] combined global and local VFM procedures to locate impact damage in composite plates and regions near weld zones [9 - 11]. Segmented VFM was used by Hagman et al. [12] on a different paperboard material to identify stiffness in discrete regions and by Kretschmann et al. [13] in wood stiffness identification. Results of a numerical simulation of a solid rocket propellant with smoothly varying modulus has been examined using VFM, leading to a polynomial approximation for modulus [14]. Zhang et al. [15] used manual virtual fields to identify mechanical properties of optic nerve tissues. They also compared analysis speed with another inverse method, finite element modelupdating (FEM-U), and found that VFM was 125 times faster than FEM-U. Bersi et al. [16] examined regional, anisotropic, nonlinear variations of mice aortas under pressure using VFM; results compared favorably with those properties identified from biaxial tests. VFM has been used to identify hightemperature response of 304 stainless steel [17] and rubbers at medium strain rates [18]. Nguyen et al. 
[19] extended VFM to enforce equilibrium in Fourier space, called F-VFM, which is especially appropriate for smoothly varying heterogeneous stiffness identification.

In this work, the emphasis is placed on identification of smoothly varying, and thereby heterogeneous, stiffness. To motivate confidence in the stiffness identification of a material containing heterogeneity, a simple specimen geometry and loading configuration were employed, namely a dogbone tensile specimen loaded in uniaxial tension. Successful identification with this simple geometry will allow some direct comparison with strain profiles, which may not be possible in more complex stress states. In practice, heterogeneous stiffness identification will likely employ a unique specimen geometry and a unique applied loading configuration. Finally, formulations that allowed stiffness identification without a priori knowledge of heterogeneity location, size or geometry were utilized.

The material chosen for evaluation was paperboard, an orthotropic composite material. Paperboard is unique within the scope of composite materials because it is composed almost entirely of cellulose fibers (some inorganic fillers or recycled material residue may be present), making them the reinforcing fiber and supporting matrix. The variety of paperboards is quite large because no two papermachines can produce the same paperboard given the same pulp. This characteristic is often called the "signature" of the papermachine. Even within a particular papermachine, the paperboard can change from hour-to-hour because of varying source material. Koning [20] provides a comprehensive list of paperboard grades, including pulping process, stock preparation, papermachines and nominal properties. Paperboard heterogeneity is acknowledged in the paper physics community, "Uniformity of quality depends on uniformity of structure" [21]. Sources of variability include mass variation [22], local fiber orientation [23, 24], fiber length distribution, and nonuniform intra-fiber bonding [25]. Size of nonapparent heterogeneities in paper materials have been estimated from $\operatorname{order}(\mathrm{mm})[26,27]$ to $\operatorname{order}(\mathrm{cm})[28,29]$.

Two FEM simulations were performed to examine the effect of stiffness identification on a model containing nonuniform, nonsymmetric stiffness heterogeneities. Experimentally, DIC was used to determine the in-plane strains of two different paperboard specimens under uniaxial tensile stress. Besides the homogeneous stiffness identification, two other VFM formulations were used to identify nonuniform stiffness. Success of stiffness identification was evaluated with an additional VFM formulation designed to examine local material equilibrium. A polyethylene terephthalate (PET) specimen was also tested to demonstrate homogeneous behavior.

\section{Methods and Materials}

\section{Application of VFM to Heterogeneous Materials}

Four different formulations of VFM were used in this work and are described here. A more detailed de- 
velopment of VFM is given by Pierron and Grédiac [1]. VFM is based upon the principle of virtual work (PVW) and, for plane stress problem in the absence of body forces, can be written as

$$
\int_{S}\left(\sigma_{x} \varepsilon_{x}^{*}+\sigma_{y} \varepsilon_{y}^{*}+\sigma_{s} \varepsilon_{s}^{*}\right) d S=\int_{\partial S} \bar{T}_{i} u_{i}^{*} d l
$$

where $S$ is the area of 2-D domain, $\sigma_{i}$ are stresses within $S, u_{i}^{*}$ are VFM admissible (continuous and differentiable) virtual displacements, $\varepsilon_{i}{ }^{*}$ are virtual strains associated with $u_{i}^{*}, \bar{T}_{i}$ are tractions applied on boundary of $S$, and $\partial S$ is the portion of $S$ over which $\bar{T}_{i}$ are applied.

A uniaxial tensile test of a homogeneous linear elastic orthotropic material performed in the $y$ direction (cross-machine direction in this work) provides only information to identify $Q_{22}$ and $Q_{12}$ because $\sigma_{6}=0$. Although $Q_{66}$ is an independent material property for orthotropic materials, a uniaxial tensile test will not activate sufficient $\sigma_{6}$ for its identification. Therefore, we used a linear elastic isotropic constitutive equation given by

$$
\left\{\begin{array}{l}
\sigma_{x} \\
\sigma_{y} \\
\sigma_{s}
\end{array}\right\}=\left[\begin{array}{ccc}
Q & v Q & 0 \\
v Q & Q & 0 \\
0 & 0 & \frac{1-v}{2} Q
\end{array}\right]\left\{\begin{array}{l}
\varepsilon_{x} \\
\varepsilon_{y} \\
\varepsilon_{x}
\end{array}\right\}
$$

Using Equation (2) in Equation (1) gives an experimentally useful PVW equation as

$$
\int_{S} Q\left[\left(\varepsilon_{x} \varepsilon_{x}^{*}+\varepsilon_{y} \varepsilon_{y}^{*}+\frac{1}{2} \varepsilon_{s} \varepsilon_{s}^{*}\right)+v\left(\varepsilon_{x} \varepsilon_{x}^{*}+\varepsilon_{y} \varepsilon_{y}^{*}-\frac{1}{2} \varepsilon_{s} \varepsilon_{s}^{*}\right)\right] d S=\int_{\partial S} \bar{T}_{i} u_{i}^{*} d l
$$

In many test geometries, the full-field displacements are measured, from which $\varepsilon_{i}$ are determined, and the resultant external loads, $\bar{T}_{i}$, are measured. An important development of VFM was optimized, piecewise virtual fields that were continuous, differentiable and optimized to provide the lowest noise sensitivity for all stiffness components [1]. They are called piecewise because $u_{i}^{*}$ need only be $C^{0}$ continuous and so a VF mesh is used to create the necessary virtual fields. In practice, Equation (3) is discretized using summations and two $u_{i}^{*}$ are selected for identification of $Q$ and $v$. Finally, Equation (3) represents the first VFM formulation and was used for homogeneous stiffness identification.

The second VFM formulation modifies Equation (3) to examine heterogeneity. Discrete heterogeneity can be examined by dividing the specimen into different subregions, often based on some a priori knowledge of heterogeneity or some other criteria. Each subregion is assumed to be itself homogeneous, and the sum of the subregions must equal the entire region $S$. Dividing $S$ into three subregions, for example, $S^{(1)}, S^{(2)}$, and $S^{(3)}$ gives a new VFM equation as 
Equation (4) allows the identification of six constitutive parameters. Although many subregions could be defined, several practical limitations arise, and foremost is the need for sufficient strain information. Problems begin to occur when the subregions are too small or when the subregion contains low magnitude strains as compared to other subregions. Practical usage has shown that special care is needed in the identification process when there are six (or more) subregions. Note that it is not required that the subregions be contiguous. Equation (4) represents the second VFM formulation used and was denoted segmented VFM.

The third VFM formulation was a contribution from Nguyen et al. [19] where the solution of Equation (3) was performed in the spatial frequency domain by means of Fourier analysis. That work used a spatially varying $Q$ represented as

$$
Q(x, y)=\sum_{m=0}^{N} \sum_{n=-N}^{N} a_{m, n} \cos 2 \pi\left(\frac{m x}{L_{x}}+\frac{n y}{L_{y}}\right)+\sum_{m=0}^{N} \sum_{\substack{n=-N \\ m+|n| \neq 0}}^{N} b_{m, n} \sin 2 \pi\left(\frac{m x}{L_{x}}+\frac{n y}{L_{y}}\right)
$$

where

$x, y \quad$ : spatial coordinates in $x$-, $y$-directions

$L_{x}, L_{y} \quad: \quad$ specimen dimensions in $x$-, $y$-directions, $44 \mathrm{~mm}$ and $140 \mathrm{~mm}$, respectively

$a_{m, n}, b_{m, n}:$ Fourier coefficients of the series

$m, n:$ spatial frequency components

$N$ : maximum values of indices

As suggested by Nguyen et al. [19], the virtual fields, $u_{i}^{*}$, were unit amplitude sine/cosine waves with the identical frequencies to those for $Q(x, y)$ in Equation (5). Those virtual fields were not optimized for experimental noise. One important simplification was that $v$ is assumed to be constant. Through several trials, they found that the frequencies for $Q$ and $u_{i}^{*}$ should be identical for good stiffness identification. Therefore, higher order frequencies would be required for Poisson ratio identification, because the lower frequencies were used for stiffness identification. The examination of higher order frequencies for Pois- 
son ratio identification was beyond the scope of this work. Subsequently, we examine the effect of the assumption for a constant $v$. We denoted this third VFM formulation as F-VFM.

The fourth VFM formulation was used by Devivier et al. [8] to detect damage in composite materials by examination of local equilibrium. A similar procedure was used to examine errors in FEM modeling [30]. Discrepancies in local equilibrium are "equilibrium gaps" (EGs) and can be used to locate boundaries of regions that are not in equilibrium with each other when material homogeneity is assumed.

Equation (3) can be rewritten as

$$
\int_{S^{\prime}} Q\left[\left(\varepsilon_{x} \varepsilon_{x}^{*}+\varepsilon_{y} \varepsilon_{y}^{*}+\frac{1}{2} \varepsilon_{s} \varepsilon_{s}^{*}\right)+v\left(\varepsilon_{x} \varepsilon_{x}^{*}+\varepsilon_{y} \varepsilon_{y}^{*}-\frac{1}{2} \varepsilon_{s} \varepsilon_{s}^{*}\right)\right] d S^{\prime}=\int_{\partial S} \bar{T}_{i} u_{i}^{*} d l
$$

Equation (6) describes the equilibrium of a region $S^{\prime}$, where $S^{\prime}$ is a portion of a larger region, $S$. If both $Q$ and $v$ are known, e.g., by use of Equation (3) (or Equation (4) or with F-VFM), and $u_{i}^{*}$ are VF admissible, then summation of the integrals should be zero; a nonzero result is an "equilibrium gap" in $S$. In absence of external forces, the final term containing $\bar{T}_{i}$ is 0 . The virtual mesh used for EG analysis is shown in Figure 1. The inspection window, i.e., the region $S$, composed of the four virtual elements, is rastered, horizontally and vertically across the entire specimen of region $S$. Two parameters can be adjusted to affect EG results: the number of data points in each element and the amount of movement of the inspection window to the next location. For this work, each virtual element contained 9 DIC data points ( 3 points $x 3$ points) and the inspection window was moved by a single DIC row or column. All virtual degrees of freedom for nodes on the edge of the inspection window are set to zero, i.e., nodes 1, 2, 3, 4, 6, 7, 8, 9 are fixed. This process eliminates all work of external forces on the window boundary. Virtual node 5 is subject to a $45^{\circ}$ displacement fixed at $\left|u^{*}\right|=1$. Equation (6) is discretized using summations, and the EG contour is determined by sliding the window over the entire specimen.

Calculation of a " 0 " EG does not guarantee that $Q$ and $v$ were correctly identified. If the correct values for stiffness and Poisson ratio are correctly identified, then the EG will be zero. Alternatively, if the correct values for $Q$ and $v$ are both multiplied by an arbitrary factor $K$, the EG will also sum to zero. Although a $K$ factor is an important consideration in a homogeneous EG evaluation, concern regarding a $K$ factor is less important in heterogeneous EG evaluation because all the spatially varying stiffnesses would need to be changed by $K$, which is unlikely.

The previous formulations incorporate $Q$ and $v$ as the constitutive parameters. The following expression shows the relationship with modulus, $E$ : 


$$
Q=\frac{E}{1-v^{2}}
$$

The large amount of void space within paper materials makes it difficult to accurately measure material thickness, thus the paper industry compares materials based on specific stiffness. Measuring a paperboard stiffness in force/width units, thereby avoiding a thickness measurement, and dividing that stiffness by material weight/area, also avoiding a thickness measurement, the papermaker has determined a specific stiffness with units $\mathrm{N} \cdot \mathrm{m} \cdot \mathrm{kg}^{-1}$ or, equivalently, $\mathrm{km}^{2} \cdot \mathrm{s}^{-2}$. The latter units are preferred because they are a direct measurement from ultrasonic time of flight, i.e., $Q / \rho=V^{2}$, where $V$ is the ultrasonic velocity. When reporting identification results, specific stiffness units, $\mathrm{km}^{2} \cdot \mathrm{s}^{-2}$, will be used.

The homogeneous VFM (Equation (3)) and segmented VFM (Equation (4)) both used a regularly spaced VFM mesh that had 4 virtual elements (horizontal) x 6 virtual elements; each element covered 11 $\mathrm{mm}$ (nominal) x $23 \mathrm{~mm}$ (nominal) and is denoted by the white lines in Figure 2. Grid-like meshes are not required but were used here for simplicity.

All analyses for each of the four VFM formulations were performed in Python programming language (Python Software Foundation, www.python.org). Full field strains were determined by DIC analysis of a composite image made from a pixel average of three sequential images. Amount of strain data, determined in the DIC analysis, varied for each specimen, but was always more than 2500 data points.

\section{Materials}

The examined material was a commercial unbleached, kraft single-ply linerboard with nominal physical properties: grammage $209 \mathrm{~g} \cdot \mathrm{m}^{-2}$, thickness $0.30 \mathrm{~mm}$, density $688 \mathrm{~kg} \cdot \mathrm{m}^{-3}$ and $1.1 \%$ ash. The approximated density for cellulose is $1500 \mathrm{~kg} \cdot \mathrm{m}^{-3}$, so this material has more than $50 \%$ void space. Precise fiber composition of this material is unknown, but it contains both virgin and recycled fibers; additionally, wet strength additives and fillers are commonly present in commercial paperboard. This material was also used in other work by the authors, e.g. [31-33], and was selected because the material had behavior in those works that suggested the likelihood of heterogeneities.

The specific causes of particular heterogeneities in this material are reserved for ongoing work. Some physical heterogeneities, specifically thickness and local mass, have been successfully characterized in paper materials by twin-laser profilometry and radiography [34]. Other physical heterogeneities, changes in local fiber orientation, local bonding, local residual stresses created by de-watering, have not been characterized on "large" specimens. 


\section{Test Configuration \& Load Schedule}

Tensile tests were performed on an Instron $^{\circledR}$ (Norwood, Massachusetts) Model 5865 test machine equipped with serrated-face pneumatic grips, $50 \mathrm{~mm}$ wide. Specimens were dog-bone shaped, as shown in Figure 2. Distance between grips was $180 \mathrm{~mm}$. The load schedule for one of the tests is shown in Figure 3, and the load-displacement cycles for each specimen are shown in Figure 4. Eleven cycles, between $15 \mathrm{~N}$ and $120 \mathrm{~N}$, were performed to examine consistency of the heterogeneous strains. After five cycles, the specimen was rotated $180^{\circ}$ (top-to-bottom) and the test resumed. Rotation of the specimen was used to ensure that heterogeneities were specimen specific and not an artifact of test procedure. The upper limit of $120 \mathrm{~N}$ was selected such that the linerboard remained in the linear elastic regime and was unique to the material tested. The test sequence started with a pre-tensile force at $15 \mathrm{~N}$ held for $35 \mathrm{~s}$ followed by a load controlled sequence at $2 \mathrm{~N} \cdot \mathrm{s}^{-1}$ to $120 \mathrm{~N}$. Specimens were held at a tensile force of $120 \mathrm{~N}$ for $35 \mathrm{~s}$ after which the tensile force was reduced to $15 \mathrm{~N}$ at a rate of $-2 \mathrm{~N} \cdot \mathrm{s}^{-1}$. The $35 \mathrm{~s}$ hold period at $120 \mathrm{~N}$ was used to allow a small amount of creep to occur and deformation to stabilize. Each ramp, increasing or decreasing tensile force, and hold periods at $120 \mathrm{~N}$ were held for $35 \mathrm{~s}$. The lower hold period, at $15 \mathrm{~N}$, was held for $70 \mathrm{~s}$. After the 11th cycle, specimens were tested to failure with a grip displacement of $1 \mathrm{~mm} \cdot \mathrm{min}^{-1}$. Load and grip displacement data were collected at $0.5 \mathrm{~Hz}$. Specimens were preconditioned at 30\% relative humidity $(\mathrm{RH})$ for $24 \mathrm{~h}$ and reconditioned to $50 \% \mathrm{RH}$ for $24 \mathrm{~h}$. Because all cellulosic materials experience moisture content - $\mathrm{RH}$ hysteresis, moisture preconditioning to a lower $\mathrm{RH}$ than test environment $\mathrm{RH}$ ensures all tests were performed on the adsorption isotherm [35]. All tests were performed in a controlled environment at $50 \% \mathrm{RH}$ and $23^{\circ} \mathrm{C}$.

Figure 4 shows that the load-displacement cycles were repeatable, even after rotation of the specimen after the 5th cycle. Utmost care was used to ensure specimen alignment before each set of cycles.

The material was tested in the $y$-direction (less stiff, called the CD or cross-machine direction by those in the paper industry). Even with careful paperboard manufacturing control, some "streaking" is possible. Streaks are heterogeneities aligned with the $x$-direction (machine or running direction) of the material and can be caused by many factors, including mass differences, fiber misorientation, and wire and/or roll defects. Testing in the $y$-direction increased the likelihood of the presence of a streak in a specimen.

\section{Digital Image Correlation}

The surface of the paperboard was examined with a stereo DIC system whose details are listed in Table 1. An optimized random gray level pattern, courtesy of Bossyut [36], was produced on a sheet of the material, nominally $205 \mathrm{~mm} \times 250 \mathrm{~mm}$, using a Sharp ${ }^{\circledR}$ (Sharp Electronics Corp., Mahwah, NJ) MX$3100 \mathrm{~N}$ copier. Specimens were cut from the sheet after pre-conditioning and conditioning. The DIC pat- 
tern is shown on a specimen in Figure 5, and a close-up view is shown in Figure 6.

The main objectives of the involved tests, i.e., measuring heterogeneous strains at a relatively small strain level, are challenging from a DIC point of view. First, one needs to avoid aliasing effects because the resulting banding effects might be confused with true heterogeneous material behavior. As can be inferred from Figure 6, both white and black speckles have a pixel sampling well exceeding 3 pixels per speckle, and moreover, smooth contrast transitions are present between the white and black regions as such avoiding the increase of the interpolation bias in DIC to a maximum extent. Second, one needs to guarantee that the measured strains are true signals well beyond the noise floor. Accordingly, a dedicated signal-to-noise study needs to be performed to find an optimal compromise between smoothing and signal reconstruction.

Resolution was determined by performing the DIC analysis on two averaged images. The reference image was the average of images captured at $10 \mathrm{~s}, 12 \mathrm{~s}$ and $14 \mathrm{~s}$; the analyzed image was the average of images captured at $16 \mathrm{~s}, 18 \mathrm{~s}$, and $20 \mathrm{~s}$, where the times are measured from the beginning of cycle 1 .

Displacement and strain resolutions were an important aspect of heterogeneity examination. Subset size, step size, strain window size, interpolation order, shape function, prefiltering, among other factors, all contribute to resolution. Small resolution can be achieved by enlarging subset, increasing step size and expanding the area used for strain calculation, but such choices effectively smooth the heterogeneous strains and reduce the amount of data. Strain smoothing reduces the ability of VFM to identify peaks and valleys of stiffness heterogeneities. For F-VFM, quantity of data across the specimen width determines the maximum number of terms in the Fourier analysis and limits the likelihood of determining high stiffness gradients.

A parametric DIC analysis was performed via MatchID [37] comparing the effect of different selections and is shown in Figure 7. The choice of the parameters in Table 1 was based on the virtual strain gage (VSG), which was approximately asymptotic with minimum possible strain resolution. The VSG is defined as $\left[(S W-1)^{*}\right.$ Step $]+$ Subset and as such reflects the contribution of subset, step and strain window to the calculation of strain parameters. For the parameters examined, the minimum resolution was 38 $\mu \varepsilon$ with a VSG of 181 pixels or $6.52 \mathrm{~mm}$, which would have given only 7 data points across the specimen width. As will be shown, a strain resolution of $65 \mu \varepsilon$ could sufficiently discriminate the strains for the material examined. The parametric analysis was designed to reduce the random portion of the experimental error. One of the authors has been a part of the effort to examine both random and systemic errors [38, 39]. Here, we do not address systematic errors explicitly, but specimen size, geometry and loading procedure were chosen to reduce systematic error.

For the heterogeneity analysis effort, reference images were the average of three consecutive images captured during the first $15 \mathrm{~N}$ load portion of the test; each cycle had a unique reference image. Ana- 
lyzed images were the average of three consecutive images captured during the $120 \mathrm{~N}$ load portion of each test and are denoted by the gray markers (Cycles 1-10) and red marker (Cycle 11) in Figure 3.

\section{FEM Simulation}

Two FEM simulations were performed to extend those published in Ref [19] and examine the effect of nonregular heterogeneities in nonsquare models.

The FEM model used for both simulations is shown Figure 8. The model was $1 \mathrm{~mm}$ thick and the material had density of $1000 \mathrm{~kg} \cdot \mathrm{m}^{-3}$. The boundary conditions include $u(0 ; 0)=0$ and $v(x ; 0)=0$; uniform tensile pressure, $P$, was applied at $y=180 \mathrm{~mm}$. ANSYS ${ }^{\circledR}$ (Academic Research, Release 9.0) PLANE42 elements were used throughout. In the necked-down region, the elements were $0.5 \mathrm{~mm} \times 0.5 \mathrm{~mm}(24640$ elements), and automatic meshing was used in the upper and lower tab regions, producing a total of 34359 elements. The size of the square elements created sufficient mesh density to achieve strain convergence within the heterogeneities.

The specific isotropic stiffness of the heterogeneity used in the first simulation is shown in Figure 9. The specific stiffness was calculated from the input modulus, $E$, and the density. It is important to note that although the stiffness heterogeneity has a defined shape and orientation and is, therefore, heterogeneous, at any point within the model the stiffness is isotropic, i.e., has no preferred orientation. Instead of trying to estimate the size, location and magnitude of a heterogeneity in paperboard, which are unknown to this point, we chose a heterogeneity shape and orientation to demonstrate the capability of FVFM to identify a nonaxisymmetric, smoothly varying stiffness inclusion. The center of the inclusion is $30 \%$ stiffer than far-field material and is similar in size and stiffness gradient to that modeled by Kim et al. [6]. Multiple heterogeneities, one for stiffness and another for Poisson ratio, were used in the second simulation and are shown in Figure 10 and Figure 11. For this simulation, both stiffness and Poisson ration are isotropic (have no preferred direction anywhere in the model), while both are heterogeneous. The second simulation was used to examine the effect of a varying Poisson ratio on the F-VFM identification, which uses $v=$ constant in its implementation. The stiffness for the second simulation had the same shape and magnitude of stiffness heterogeneity as the first simulation, but in a different location. The centroids of the $Q$ and $v$ inclusions were coincident, but the orientation of the $v$ heterogeneity was rotated $90^{\circ}$ with respect to the $Q$ heterogeneity. The $v$ inclusion had a minimum value of 0.15 , while the far field material had $v=0.3$. Each inclusion was stiffer than surrounding area and so produced less strain and, therefore, reduced the sensitivity of VFM identification.

The inclusions simulated were chosen to examine the capability of F-VFM stiffness identification and, in the absence of specific knowledge of paperboard stiffness heterogeneity, were expected to be more difficult to characterize than those occurring in most paperboards. 


\section{Results Simulations}

Strains for the first simulation are shown in Figure 12 and show the presence of the centrally located inclusion in the gradients of each strain and the presence of nonzero $\varepsilon_{s}$ at the upper and lower boundaries. F-VFM identification results are shown in Figure 13 for $N=5,9,12$, and for smoothed $N=12$, where $N$ is the summation index in Equation (5). $N=12$ was the maximum possible $N$ and was limited by the specimen width. The smoothed stiffness was produced with a multiquadric Radial Basis Function (Rbf) algorithm within SciPy [40] using a smoothing parameter $=15$. Because Rbf algorithms behave as if a thin membrane were stretched over a response surface, in this case, stiffnesses near boundaries were preserved. Larger $N$ s could have been employed by interpolating to increase the number of points. Strain interpolation with larger $N$ did not improve EG results, which is discussed subsequently, for these simulations.

Some of the ringing artifacts, the spurious signals near the boundaries of the specimen, could likely be reduced by using a different stiffness expansion in Equation (5), further reducing the number of terms in the $x$-direction, the $m$-terms. Already the $x$-direction has half the terms of the $y$-direction. Because a priori knowledge of the heterogeneities was not assumed for the simulation or for the subsequent experimental work, no further reduction in $m$-terms was investigated.

Figure 14 shows the contour maps for RMS error for smoothed $N=12$, and for homogeneous and smoothed $N=12$ EG. Significant error along the vertical boundaries was visible. F-VFM overestimated the inclusion stiffness by $3.0 \%$; the homogeneous identification underestimated the inclusion stiffness by 22.2\%. The EG contours provided a good method to show the improved identification of the F-VFM as compared with the homogeneous identification. Although the region near the inclusion was visible in the heterogeneous EG, the inclusion region was much more visible in the homogeneous EG contour. For the homogeneous EG analysis, portion of the model far from the inclusion showed deceptive equilibrium. This effect was noted when EG was introduced earlier, that if $Q$ and $v$ differ by a constant $K$, the EG analysis will indicate local equilibrium. The same effect is visible in the center of the inclusion region, where a narrow equilibrium region existed. This result was beneficial for ensuing analyses; $Q$ error maps cannot be generated if the magnitude, location and geometry of the heterogeneities in a material are unknown, but EG maps provide a reliable evaluation of stiffness identification.

Strains for the second simulation are shown in Figure 15. Unsymmetrical and nonuniform strains were evident. Due to the model and load application, $\varepsilon_{y}$ has the large magnitude, but the Poisson inclusion is hardly visible in the $\varepsilon_{y}$ contour map. The intersection of both inclusions is quite stiff, as indicated by the very low $\varepsilon_{x}$ in that location. Identification for second simulation is shown in Figure 16. Error and equilibrium gap results for second simulation are shown in Figure 17. Contour error was predominantly in the Poisson heterogeneity because the stiffness identification compensated for the enforced homogeneous 
Poisson ratio in the F-VFM formulation. The RMS error for the intersection of the inclusions was 2.1\% too high, whereas the homogeneous error was $22.8 \%$ too low. As in the first simulation, EG contours provided more information. For the homogeneous identification, the EG showed large nonequilibrium around the stiffness heterogeneity because $Q$ was an order of magnitude larger than $v$; the $v$ heterogeneity showed greater degree of equilibrium. Compared to the homogeneous EG, the heterogeneous EG analysis indicated more equilibrium in the $Q$ heterogeneity and less in the $v$ heterogeneity.

Table 2 lists the error and EG results for both simulations. Because the stiffness distribution within the simulated specimen is not normal and, therefore, the error and EG contours are not normal, percentiles were used to evaluate the data. Care should be exercised when using conventional statistical metrics when comparing results. Error results proved incapable of providing meaningful parameters to rank the identifications. Homogeneous results had the lowest 90th, 50th, and 10th percentile errors. The 10th percentile is the value below which $10 \%$ of the data will be found. This apparent small error occurred because the area of the simulated specimen with stiffness greater than $10 \%$ of the mean stiffness was only $7.1 \%$ of the total area. The errors for the heterogeneous and smoothed heterogeneous identifications were larger because of the "ringing" artifacts along the vertical boundaries of the specimen, as seen by the high stiffness on those edges in Figure 13 and Figure 16.

For each simulation, the homogeneous EG analysis indicated deceptive equilibrium away from the inclusions. This effect was noted when EG was introduced earlier, that if $Q$ and $v$ differ by a constant $K$, the EG analysis will indicate local equilibrium. The same effect is visible in the center of the inclusion region, where a narrow equilibrium region existed.

The simulated inclusions were well characterized, i.e., relative size, location and stiffness magnitude, by F-VFM. The largest errors existed well away from the inclusions where the high frequencies needed to represent the inclusions were not damped. These results indicated that F-VFM was capable of stiffness identification for simulated inclusions more dramatic than we expect to assess in paperboard.

\section{Paperboard}

Two paperboard specimens were tested per the load schedule shown in Figure 3. The $\varepsilon_{y} / \operatorname{mean}\left(\varepsilon_{y}\right)$ for each cycle is shown in Figure 18. Some differences were apparent between cycles 5 and 6, when the specimen was rotated $180^{\circ}$. A small amount of local softening of the specimens was evident, where the magnitude and size of region covered by the high strain were gradually increased. So, although the macroscopic strain indicated that the specimen remained in the linear elastic region, the local strains showed some nonlinearity.

Figure 19 shows the mean $\left(\varepsilon_{y}\right)$ and $\operatorname{COV}\left(\varepsilon_{y}\right)$ for each cycle. The rotation of the specimens after cycle 5 did not have a large effect on mean $\left(\varepsilon_{y}\right)$, but $\operatorname{COV}\left(\varepsilon_{y}\right)$ was affected. For specimen 1 , the $\operatorname{COV}\left(\varepsilon_{y}\right)$ went from $10.9 \%$ to $12.2 \%$ for cycles 5 and 6 ; for specimen 2 , the change was from $9.1 \%$ to $10.1 \%$. The chang- 
1

es of the $\operatorname{COV}\left(\varepsilon_{y}\right)$ were small but readily apparent in Figure 18. We offer no definitive explanation for this behavior, but it is possible that the asymmetric loading (bottom grip fixed and moving top grip) of a heterogeneous specimen may contribute to this phenomenon.

The strain contour maps for cycle 11 are shown in Figure 20. The strain heterogeneity is apparent, and the $\varepsilon_{y}$ magnitude was two orders larger than the strain resolution listed in Table 1, which suggests that resolution was capable of discerning heterogeneity. Two different types of heterogeneity were present in the $\varepsilon_{y}$ contours: specimen 1 was dominated by a heterogeneity causing low strain, and specimen 2 by high strain. In specimen 2 , the highest Poisson strain generally coincided with high $\varepsilon_{y}$ region. The low $\varepsilon_{y}$ region in specimen 1 did not appear to have constant Poisson strain. Examination of Poisson strain is important because F-VFM assumes a homogeneous $v$. Shear strains in the $\varepsilon_{s}$ contours were small and did not appear to have high or low strain regions associated with those in the other strain contours.

As a demonstration that the DIC system and parameters would not produce a false positive result, i.e., measure heterogeneous strains in a homogeneous material, a polyethylene terephthalate (PET) specimen, $0.25 \mathrm{~mm}$ thick, of same geometry as the paperboard specimens and printed with same pattern, was examined under uniaxial tensile stress and analyzed with the same DIC analysis. The $\varepsilon_{y}$ contour map for the PET specimen is shown in Figure 21. For the PET specimen, the $\operatorname{COV}\left(\varepsilon_{y}\right)$ was 2\%, demonstrating the expected strain homogeneity and validating the selection of the DIC parameters.

Table 3 details the identification information for the four VFM analyses performed for each specimen. The homogeneous results list results from two calculations; for mean $(\varepsilon), Q=P /$ mean $\left(\varepsilon_{y}\right)$ and $v=$ mean $\left(\varepsilon_{x}\right) /$ mean $\left(\varepsilon_{y}\right)$ and homogeneous VFM (Equation (3)). Each section in the Table 3 gives the 90th, 50th, and 10th percentiles of EG along with the standard deviation EG results. The final columns list the Voigt and Reuss calculations for $Q$ and are included to represent upper and lower $Q$ bounds for a material composed of isotropic components. The Voigt and Reuss stiffnesses are

$$
\begin{gathered}
Q^{\mathrm{Voigt}}=\sum_{i=1}^{n} f_{i} Q_{i}=\operatorname{mean}\left(Q_{i}\right) \\
Q^{\text {Reuss }}=\left(\sum_{i=1}^{n} \frac{f_{i}}{Q_{i}}\right)^{-1}
\end{gathered}
$$

where $i$ is a single data point, $n$ is the total amount of data, $f_{i}$ is the area fraction of $i$ and $Q_{i}$ is the stiffness of $i$. Since all $i$ are the same size, the DIC subset size, $Q^{\mathrm{Voigt}}$ reduces to the mean $\left(Q_{i}\right)$. 


\section{Segmented VFM}

Selection of the divisions for the segmented VFM analysis (Equation (4)) relied on EG analysis (Equation (6)). A total of 171 division arrangements were evaluated where the divisions were based on the magnitude of local equivalent Von Mises strain, $\varepsilon_{\text {eq }}$, for cycle 11 , where $\varepsilon_{\text {eq }}$ is

$$
\varepsilon_{\mathrm{eq}}=\frac{2}{3}\left[\frac{3}{2}\left(\varepsilon_{x}^{2}+\varepsilon_{y}^{2}+\varepsilon_{s}^{2}\right)\right]^{\frac{1}{2}}
$$

Other cycles were also examined and gave similar divisions. EG analyses were performed by varying the lower division from the 5 th percentile of $\varepsilon_{\text {eq }}$ to the 90 th percentile of $\varepsilon_{\text {eq }}$, while also varying the upper division from the 10 th percentile of $\varepsilon_{\text {eq }}$ to the 95 th percentile of $\varepsilon_{\text {eq }}$ in $5 \%$ increments, with the constraint that the lower division was less than the upper division. For example, the initial EG analysis divided the specimen into the 5 th percentile of $\varepsilon_{\text {eq }}$ and the 10 th percentile of $\varepsilon_{\text {eq }}$, giving three divisions, from $0 \%-5 \%$, $5 \%-10 \%$ and $10 \%-100 \%$. The division of the specimen with the smallest standard deviation of the EG was selected as the "best" segmentation for the specimen.

To compare stiffness for each VFM analysis, the $Q$ percentiles for F-VFM (and smoothed F-VFM) were selected to coincide with the segmented VFM divisions. For example, if the segmented VFM divisions equally divided the specimen at $33 \%$ and $66 \%$ of $\varepsilon_{\text {eq }}$, the $82.5 \%, 50 \%$ and $17 \%$ of F-VFM $Q$ were calculated for comparison. The segmented VFM $Q$ for the $33 \%$ of $\varepsilon_{\text {eq }}$ would compare with the $82.5 \%$ of F-VFM $Q$; the low strain region selection for $\varepsilon_{\text {eq }}$ corresponds to the high stiffness portion of F-VFM $Q$. On the other end of the distribution, the center point of $66 \%$ of $\varepsilon_{\text {eq }}$ is $83 \%$ of $\varepsilon_{\text {eq }}$, which should be related to the lowest $17 \%$ of $Q(100 \%-83 \%)$. The $83 \%$ percentile is the value below which $83 \%$ of the observations may be found or, conversely, $17 \%$ of the data are above $83 \%$.

None of the strain components was normally distributed, as an example the $\varepsilon_{\text {eq }}$ distribution is shown in Figure 22, where the divisions for the best EG analysis are also given. Because they are not normally distributed, care must be used when evaluating the results. For this reason, four different metrics for each EG analysis were used to represent the distribution. The $90 \%$ and $10 \%$ were used to portray the range of EG; $50 \%$ was the range median; standard deviation was used to describe the scatter. By reviewing each of these metrics the improvement in the quality of the identification can be described. The segmented divisions for each specimen in Figure 23 shows the noncontiguous scatter of material regions within each $\varepsilon_{\text {eq }}$ range.

\section{F-VFM Identification}

The $\varepsilon_{y}$, F-VFM $Q$ and smoothed F-VFM $Q$ for each specimen are shown in Figure 24. The maximum possible $N$ using noninterpolated strain data was $N=7$. The smoothed $Q$ was produced with a multi- 
quadric Radial Basis Function (Rbf) algorithm using a smoothing parameter of 15. The gray horizontal lines indicate the nominal vertical median of the failure location; the failure zone for each specimen was approximately $\pm 3 \mathrm{~mm}$ of the indicated failure location.

Although only two specimens are presented here, their failures represent two of the common failure occurrences. In specimen 1, the failure zone occurred in a gradient region, between low and high stiffness zones. In specimen 2, the failure zone occurred directly in the low stiffness zone, near the specimen's right vertical edge, that did not span the specimen width.

As given in Table 3, EG results were generally ranked from best to worst: smoothed F-VFM, FVFM, segmented VFM and homogeneous VFM. For each specimen, the EG analysis for smoothed FVFM had the smallest range, the second smallest $50 \%$, and the smallest standard deviation.

\section{Comparison of $\boldsymbol{Q}$ Identification}

To compare $Q$ for each VFM analysis, the $Q$ percentiles for F-VFM (and smoothed F-VFM) were selected to coincide with the segmented VFM divisions. For specimen 1, the division of the highest $85 \%$ of $\varepsilon_{\text {eq }}$ means that division included all the strains from the $100 \%-85 \%$, the mean was $92.5 \%$. Because high strain indicates low stiffness, the 7.5\%-tile of F-VFM $Q$ was selected for that division. The mean of the middle-segmented division was $67.5 \%$, midpoint between $85 \%$ and $50 \%$, so the $32.5 \%$ F-VFM $Q$ was selected. Finally, the lower $50 \%$ division has a mean of $25 \%$ of $\varepsilon_{\text {eq }}$, so the $75 \%$ F-VFM $Q$ was selected. The same calculations were made for specimen 2. Because the divisions for segmented VFM identification were different for each specimen, the \%-tiles for F-VFM were changed to correspond.

Identification of Poisson ratio for the segmented VFM analysis indicated the practicality of using the F-VFM analysis. Recall the Simulation 2 reduced $v$ from 0.3 in the greater portion of the specimen to 0.15 in the center of the inclusion. Even so, the RMS error for stiffness was small near the Poisson inclusion. For the paperboard specimens, the variation of Poisson ratio is smaller than in the simulation. For specimen $1,85 \%$ of area had $v=0.15-0.17$, whereas for specimen $2,85 \%$ of the area had $v=$ $0.16-0.20$.

For both specimens, the segmented VFM identified a greater stiffness in the low Von Mises strain region than either F-VFM analyses, whereas both F-VFM analyses identified a smaller stiffness than the segmented VFM in the less stiff region. For specimen 1, the segmented VFM had a smaller EG range, median and standard deviation than the homogeneous VFM analysis; for specimen 2, the segmented VFM EG analysis also had a smaller range, standard deviation and a smaller median EG than the homogeneous analysis. Even if all the EG metrics for a segmented analysis are similar to a homogeneous analysis, the segmented analysis provides a range of stiffness values whose equilibrium is improved 
within the divisions.

\section{Discussion}

One benefit of the EG contour for the homogeneous analyses of the simulations was the obvious presence of the inclusions shown in Figure 14 and Figure 17, and the contour clearly showed regions where equilibrium was violated. The stiffness contours (Figure 13 and Figure 16) also showed the inclusions and provided further verification of inclusion magnitude and location from an unrelated (EG vs F-VFM) analysis.

Although the stiff inclusions in the simulations seemed to be well identified when viewing the contour maps, the statistical analysis (Table 2) did not provide demonstrable success of the F-VFM identification. The stiffest $90 \%$ of the inclusion in the 1 st simulation comprised $7.1 \%$ of the model area, less than the $10 \%$ values in Table 2. For each analysis, the presence of the Poisson heterogeneity increased the EG metrics, spans from $90 \%$ to $10 \%$ increased and standard deviation increased, whereas the error metrics were relatively unchanged. Therefore, the $v=$ constant assumption of the F-VFM formulation should be complemented with other tools capable of examining possible Poisson variation.

For the paperboard analysis, the other tool employed was the segmented VFM analysis. By dividing the specimen into different regions based on Von Mises strain, $\varepsilon_{\mathrm{eq}}$, and using EG analysis, the material has regions with improved equilibrium. However, the borders between segments will not be in equilibrium, because the stiffness was smoothly varying and the borders represent an abrupt change in identified stiffness and Poisson ratio. Because the EG window is $6 \times 6$ data points (each element is $3 \times 3$ data points), the segment borders had nonequilibrium within 6 data points, vertically and horizontally. The segmentation increases nonequilibrium locally, so when the segmented VFM EG metrics were similar to the homogeneous analysis, the identification results are considered to reflect local constitutive behavior. In a few locations for each specimen, the segment was smaller or very similar to the size of the EG window. In those locations, the EG analysis will indicate nonequilibrium. More elaborate segmentation algorithms could be developed, but based on EG analysis, one component of the algorithm development would a minimum size restriction on a material region, such that it would be larger than the EG window.

The value of Von Mises strain as a tool to segment the specimens is demonstrated by the improvement in size of EG range and std(EG) for the homogeneous and segmented VFM analyses. For specimen 1, the EG range (difference between $90 \%$ and 10\% values) went from 210.8 to 157.6 and the std(EG) went from 87.6 to 63.3. Similar improvement was found in specimen 2, where the range was reduced from 177.2 to 138.3 and the $\operatorname{std}(E G)$ from 66.8 to 55.2 . Because range, $90 \%$ to $10 \%$, represents $80 \%$ of the material, a large portion of the specimen had improved equilibrium using segmentation.

Histograms for the $\varepsilon_{\text {eq }}$ shown in Figure 22 are bi-modal and had a large range, especially considering 
that the tensile test primarily activates $\varepsilon_{y}$. Specimen 1 had a large region of low strain and was left-tailed, which indicated small regions of high strain, whereas specimen 2 had an abrupt cutoff of high strain, but was right-tailed. The localized high strain, see the upper portion of specimen 1 in Figure 19, represents a challenge for DIC investigation and it was likely that peak $\varepsilon_{y}$ was underestimated. Similarly, the localized low strain in specimen 2 was likely overestimated. Regardless, the segmented VFM effectively smoothed the stiffness variation in those regions. The underestimation of $\varepsilon_{y}$ helps explain the higher minimum stiffness of the segmented VFM when compared with the F-VFM, especially since the low strain region extended across the width for both specimens. The contours of stiffness in Figure 24 show a localized high stiffness region for both specimens, each covering less than half the specimen width. The lack of data across the specimen, as compared to the length, reduced the number of coefficients allowed for the F-VFM analysis, reducing the capability to identify a peak stiffness, exacerbating the overestimation of strain in low strain regions.

Another issue related to strain was the presence of some nonlinearity in each specimen at the loads employed here. Strains in linear elastic materials have small magnitudes, as compared to those in plasticity for instance, and deviation from linear elasticity requires resolving a fraction of an already small quantity. Determination of the nonlinearity in terms of location, size and orientation is challenging for DIC and any full-field technique. Here, the length of the VSG was $3.64 \mathrm{~mm}$ (Table 1). Accurate measurement of the nonlinearity would require the scale of the feature to be at least the size of the VSG. Strains calculated across nonlinear and linear features would be smoothed. Reduction in size of the VSG reduces resolution (decreases signal to noise) and reduces confidence in strain measurement. VFM is well-suited to identify stiffness in homogeneous nonlinear materials [15-18], but identification in locally nonlinear materials requires strain measurements at the limits of current technology.

Other nonlinearity is likely due to release of dried-in stresses. During papermaking, the cellulose fiber/water mixture is initially $99 \%$ water. By gravity drainage, suction and pressing, the web quickly reaches $40 \%$ solids (cellulose and additives), at which point the web is restrained from CD (the direction tested here) shrinkage, producing dried-in tensile stresses. Release of dried-in stresses, which are heterogeneous, occurred during the load cycling and that contributed to the selection of cycle 11 for strain measurement and stiffness identification.

Lower stiffness bounds were better identified by F-VFM. Referring to the smoothed values in Table 3 , the lower bounds for specimens 1 and 2 were $2.78 \mathrm{~km}^{2} \cdot \mathrm{s}^{-2}$ and $3.24 \mathrm{~km}^{2} \cdot \mathrm{s}^{-2}$, respectively, both lower than for the segmented VFM high $\varepsilon_{\text {eq }}$ division for each specimen. The F-VFM formulation is enforcing equilibrium in spatial frequency space, thereby incorporating the strain gradient. In Figure 24, the strain and stiffness correspond more closely in the gradient regions than in the regions of high strain. In practice, regions of high stiffness are not an issue for papermakers. Rather, it is the regions of high strain and 
low stiffness that reduce convertibility of the material. The ability of the F-VFM to identify regions of low stiffness and their corresponding magnitude can provide meaningful lower bounds of stiffness.

Because the differential strain was determined between $120 \mathrm{~N}$ and $15 \mathrm{~N}$ and was used for identification of stiffness, the identified stiffness was the secant stiffness between those load levels. For a nonlinear material, secant stiffness is higher than tangent stiffness. In this work, the identification of secant stiffness, the smoothing of high strains, which reduces peak strains, the lack of knowledge of the precise spatial variation of stiffness (for F-VFM) or the stiffness smoothing of segmented VFM combine to increase the lower stiffness bounds. Conversely, these factors also lower the upper stiffness bounds.

Comparing the lower stiffness bounds determined by smoothed F-VFM with the homogeneous stiffness determined by VFM, the lower bounds were $77 \%\left(2.78 \mathrm{~km}^{2} \cdot \mathrm{s}^{-2} / 3.60 \mathrm{~km}^{2} \cdot \mathrm{s}^{-2}\right)$ and $79 \%\left(3.24 \mathrm{~km}^{2} \cdot \mathrm{s}^{-}\right.$ ${ }^{2} / 4.11 \mathrm{~km}^{2} \cdot \mathrm{s}^{-2}$ ) of the homogeneous stiffness. Although only two specimens were examined, the consistency of the lower stiffness bounds as related to homogeneous stiffness indicates a possible papermachine signature, a unique feature introduced in processing before the paperboard reaches $40 \%$ solids at which fiber network consolidation is defined.

It was not surprising that the Voigt and Reuss bounds listed in Table 3 were not meaningful. Those bounds assume a uniform distribution of low and high stiffness materials that was not present in these specimens. In fact, the Voigt-Reuss ranges for the segmented VFM, F-VFM and smoothed F-VFM did not contain the homogeneous VFM identification.

The horizontal gray lines in Figure 24 represent the approximate tensile failure location for each specimen. Most often the failure location of paper materials has been associated with regions of high variation of local mass [41] or low mass [42]. Use of local mass in the methodology described here is part of our future work. Here we have used a global density to calculate specific stiffness. Future work will use local density. So we cannot specify that failure was caused by some issues related to mass or to other factors, like reduced bonding or local fiber misorientation. The early identification of failure zone can simplify forensic examination and provide information leading to development of improved failure mechanisms.

As anticipated, the simulations represented a more challenging heterogeneity than we found in the two specimens. The capability of F-VFM to identify location, shape and magnitude of the simulated heterogeneity provided confidence when examining paperboard. In particular, the paperboard heterogeneities were not uniform across the specimen width or well-aligned with any specimen boundaries. Both types of heterogeneities identified in the paperboard are indicative of an issue that is an ongoing concern for papermakers - streaking. That we have demonstrated both high- and low-strain streaking is significant to the industry, especially that failure occurred near streaks in both specimens. 
To this point we have relied on simulation, EG results and comparison with a homogeneous material to demonstrate the validity of our approach for smoothly varying heterogeneous stiffness identification. The large variation of stiffness found here is not unusual in paper materials. In copy paper, Yoshihara and Yoshinobu [43] found the coefficient of variation of $E$, elastic modulus, to be greater than $20 \%$ in the CD. With a similar width, but much shorter specimen, Hagman et al. [29] found a similar stiffness variation. Koreteoja et al. [44] performed 4000+ tensile tests on a paperboard material and determined that $\max (E) /$ mean $(E)$ ranged from 1.2 to 3.1 and $\min (E) /$ mean $(E)$ ranged from 0.1 to 0.7 .

The most obvious limitation of this work is the uniaxial load and specimen configuration. However, identification of stiffness heterogeneity in this simple stress state has not been presented, although several authors have demonstrated uniaxial strain heterogeneity, e.g. [25, 29]. Because paperboard is an orthotropic material, and potentially locally anisotropic, a more complex loading configuration is required to activate all strains locally for local stiffness identification. Competition between sufficient strain resolution and magnitude, along with local nonlinear strains, will pose particular problems when trying to identify a full anisotropic stiffness matrix, where components become nonlinear at different stress levels.

However, the results of this work have immediate and practical applications in the paperboard and structural paper industries. The paperboard material examined here is most often used as inner and outer facing of corrugated board. In practice, the top-to-bottom compression strength of corrugated boxes is the primary factor used to price the material. Due to the manufacturing of the material, the compression load on the boxes is in the same material direction examined here, the weaker, less stiff direction. Additionally, the most common box failure is by side panel buckling. Because stiffness is the controlling factor for buckling, knowledge of local less-stiff regions may help explain why large safety factors are necessary in box design, especially since safety factors increase material costs. Local stiffness reduction is also of importance for sack papers, where uniform stretch is desirable. If the global stretch of the material is actually dominated by regions of high local stretch, then tensile failure is likely to occur in those regions. This analysis can help identify those regions and help determine papermachine processes that contribute to the heterogeneities.

\section{Conclusion}

The objective of this work was to create a methodology to identify the location and magnitude of smoothly varying stiffness heterogeneities. Four different VFM formulations were presented and used to identify smoothly varying stiffness heterogeneity in two simulations and the actual tensile tests of two paperboard specimens. The simulations showed that because Poisson variation was a lower order effect than stiffness variation, a constant $v$ assumption still provided acceptable heterogeneous stiffness identi- 
fication. Using a local equilibrium approach, segmented VFM and F-VFM had more accurate identification than the homogeneous identification. A PET plastic specimen of same geometry as the paperboard specimens was tested to demonstrate homogeneous behavior and validate selected DIC parameters.

One type of material segmentation was presented based on Von Mises equivalent strain. The segmentation was optimized based on local equilibrium. The segmentation algorithm allowed noncontiguous regions with no limitations on segment area. Although the algorithm was sufficient for this work, one suggestion for improvements in the segmentation algorithm is to include a lower limit on region size such that it is larger the equilibrium inspection region.

Lack of DIC information across tensile specimen width limited the number of Fourier coefficients in the F-VFM formulation when a larger number of coefficients would have improved identification in high stiffness gradient regions. However, even with no a priori knowledge of the heterogeneous stiffness, the F-VFM formulation could identify a smoothly varying stiffness such that local equilibrium was improved from the homogeneous assumption.

Both segmented VFM and F-VFM provided information on the location and magnitude of smoothly varying stiffness heterogeneities. The EG analysis showed the improvement of global equilibrium of each analysis over the homogenous assumption.

This work provides a methodology to probe heterogeneous materials and assess the capability of different VFM techniques. Although the methodology was applied to a material with manufacturingcreated heterogeneities, it is applicable to damage assessment and as part of an off-line process control system. 


\section{References}

[1] F. Pierron, M. Grédiac. The Virtual Fields Method: Extracting Constitutive Mechanical Parameters from Full-Field Deformation Measurements. Springer, 2012.

[2] S. Avril, J. M. Huntley, F. Pierron, D.D. Steele. Exp. Mech. 2008. 48(4):479-494, doi:10.1007/s11340-008-9128-2.

[3] J. A. Bucaro, A. J. Romano, P. Abraham, S. Dey. J. Acoust. Soc. Am. 2004. 115(1):201-206, doi:10.1121/1.1631936.

[4] A. J. Romano, J. A. Bucaro, J. F. Vignola, P. B. Abraham. J. Acoust. Soc. Am. 2007. 121(5):26672672, doi:10.1121/1.2715459.

[5] A. J. Romano, J. A. Bucaro, S. Dey. J. Acoust. Soc. Am. 2008. 124(2):918-925, doi:10.1121/1.2945706.

[6] J. H. Kim, F. Pierron, M. R. Wisnom, K. Syed-Muhamad. Compos. Part A-Appl. S. 2007. 38(9):2065-2075, doi:10.1016/j.compositesa.2007.04.006.

[7] J.-H. Kim, F. Pierron, M. R. Wisnom, S. Avril. Compos. Part A - Appl. S. 2009. 40(12):1961 - 1974, doi:10.1016/j.compositesa.2009.09.024.

[8] C. Devivier, F. Pierron, M. R. Wisnom. Compos. Part A - Appl. S. 2013. 48:201-218, doi:10.1016/j.compositesa.2013.01.011.

[9] M. A. Sutton, J. H. Yan, S. Avril, F. Pierron, S. M. Adeeb. Exp. Mech. 2008. 48(4):451-464, doi:10.1007/s11340-008-9132-6.

[10] G. Le Louëdec, F. Pierron, M. A. Sutton, A. P. Reynolds. Exp. Mech. 2013. 53(5):849-859, doi:10.1007/s11340-012-9679-0.

[11] G. Le Louëdec, F. Pierron, M. A. Sutton, C. Siviour, A. P. Reynolds. J. Dyn. Behav. Mat. 2015. 1(2):176-190, doi:10.1007/s40870-015-0014-6.

[12] A. Hagman, J. M. Considine, M. Nygårds. Conference Proceedings of the Society for Experimental Mechanics Series, 2016. doi:10.1007/978-3-319-42255-8_20.

[13] D. E. Kretschmann, J. M. Considine, F. Pierron. Conference Proceedings of the Society for Experimental Mechanics Series, 2016. doi:10.1007/978-3-319-22449-7_8.

[14] Y. Sato, S. Arikawa, S. Yoneyama. International Conference on Experimental Mechanics, 2014. doi:10.1117/12.2081089.

[15] L. Zhang, S. G. Thakku, M. R. Beotra, M. Baskaran, T. Aung, J. C. H. Goh, N. G. Strouthidis, M. J. A. Girard. Biomech. Model. Mechanobiol. 2016. doi:10.1007/s10237-016-0858-2.

[16] M. R. Bersi, C. Bellini, P. Di Achille, J. D. Humphrey, K. Genovese, S. Avril. J. Biomech. Eng. 2016. 138(7):071005-071005, doi:10.1115/1.4033674.

[17] G. Valeri, B. Koohbor, A. Kidane, M. A. Sutton. Opt. Lasers Eng. 2017.91:53-61, doi:10.1016/j.optlaseng.2016.11.004.

[18] S.-H. Yoon C. R. Siviour. J. Dyn. Behav. Mat. 2017. 3(1):12-22, doi:10.1007/s40870-016-0090-2.

[19] T. T. Nguyen, J. M. Huntley, I. A. Ashcroft, P. D. Ruiz, F. Pierron. Int. J. Numer. Meth. Eng. 2014. 98(12):917-936, doi:10.1002/nme.4665.

[20] J. W. Koning. Corrugated Crossroads: A Reference Guide for the Corrugated Containers Industry. TAPPIPress, 1995. ISBN 0898522994. 
[21] J. A. Bristow, P. Kolseth. Paper Structure and Properties. Marcel Dekker, 1986. ISBN 0824775600.

[22] M. B. Lyne, R. Hazell. Formation testing as a means of monitoring strength uniformity. In The Fundamental Properties of Paper Related to its Uses, Transactions of the Vth Fundamental Research Symposium, Cambridge, UK, pages 74-100. FRC, Manchester, UK, 2003. ISBN 0510457509.

[23] T. Enomae, Y.-H. Han, A. Isogai. Nord. Pulp Pap. Res. J. 2006. 21(2):253-259.

[24] S. Osaki. Tappi J. 1987. 70(2):105-108.

[25] M. J. Korteoja, A. Lukkarinen, K. Kaski, D. E. Gunderson, J. L. Dahlke, K. J. Niskanen. Tappi J. 1996. 79:217-224.

[26] B. C. Donner. An heuristic model of paper rupture. In Fundamentals of Papermaking Materials, Transactions of the XIth Fundamental Research Symposium, pages 1215-1247. 1997. ISBN 0954527216.

[27] J. M. Considine, D. W. Vahey, J. W. Evans, K. T. Turner, R. E. Rowlands. J. Compos. Mater. 2011. 46(11):1323-1334. doi:10.1177/0021998311418262.

[28] M. Ostoja-Starzewski, J. Castro. Philos. Trans. R. Soc.London, Ser. A 2003.361(1806):965-985. doi:10.1098/rsta.2003.1175.

[29] A. Hagman, M. Nygårds. Nord. Pulp Pap. Res. J. 2012. 27(2):295-304.

[30] P. Ladeveze, D. Leguillon. SIAM J. Num. Anal. 1983.20(3):485-509. doi:10.1137/0720033.

[31] D. W. Vahey, J. M. Considine. Appita J. 2010. 63(1):27-31, 41.

[32] J. M. Considine, D. W. Vahey, R. Gleisner, A. Rudie, S. R. Roscoat, J.-F. Bloch. Tappi J. 2010. 9(10):25-32.

[33] J. M. Considine, F. Pierron, K. T. Turner, D. W. Vahey. Exp. Mech. 2014. 54(8):1395-1410. doi:10.1007/s11340-014-9903-1.

[34] Y. J. Sung, D. S. Keller. Appita J. 2008. 61(1):28.

[35] V. C. Setterholm, D. E. Gunderson. Observations on Load-Deformation Testing, volume 1, chapter 4, pages 115-143. Marcel Dekker, New York, USA, 1983. ISBN 0824718472.

[36] S. Bossuyt. Proceedings of the 2012 Annual Conference on Experimental and Applied Mechanics, Volume 3: Imaging Methods for Novel Materials and Challenging Applications 2013. 239-248. doi:10.1007/978-1-4614-4235-6 34.

[37] Matchid, www.matchidmbc.com, 2017.

[38] M. Rossi, F. Pierron. Int. J. Solids Struct. 2012.49(3-4):420-435. doi:10.1016/j.ijsolstr.2011.09.025.

[39] P. Wang, F. Pierron, M. Rossi, P. Lava, O. T. Thomsen. Strain 2016. 52(1):59-79. doi:10.1111/str.12170.

[40] E. Jones, T. Oliphant, P. Peterson. SciPy: Open source scientific tools for Python, 2001. www.scipy.org.

[41] R. J. Norman. Dependence of sheet properties on formation and forming variables. In Consolidation of the Paper Web, Transactions of the IIIrdFundamental Research Symposium 1965. Volume 1, pages 269-309. ISBN 0954112636.

[42] J. M. Moffat, L. R. Beath, W. G. Mihelich. Major factors governing newsprint strength. In The Fundamental Properties of Paper Related to Its Uses, Transactions of the Vth Fundamental Research Symposium 1973. Volume 1, pages 104-127. ISBN 0510457509.

[43] H. Yoshihara, M. Yoshinobu. J. Wood Sci. 2014. 60(4):287-293. doi:10.1007/s10086-014-1398-y. 
[44] M. Korteoja, L. I. Salminen, K. J. Niskanen, M. J. Alava. Mat Sci Eng A-Struct. 1998. 248(1):173180. doi:10.1016/S0921-5093(98)00491-2.

Table 1: DIC system components and parameters

Table 2: Results for both simulations. Heterogeneous results represent $N=12$ in Equation (5). EG has units specific stiffness/area $\left(\mathrm{s}^{-2} 10^{17}\right)$. Smoothing factor for $\mathrm{Rbf}=100$.

Table 3: Results of VFM analyses for the tensile specimens; $Q$ has specific stiffness units $\left(\mathrm{km}^{2} \cdot \mathrm{s}^{-2}\right)$; EG has units $\left(\mathrm{s}^{-2} \cdot 10^{22}\right)$ 
Figure 1: Schematic of equilibrium gap mesh, which was rastered throughout inspection region.

Figure 2: Paperboard specimen geometry, approximately to scale. Area inspected by VFM is filled in gray. White lines within inspection region represent VFM mesh for homogeneous and segmented heterogeneous identification.

Figure 3: Load sequence for each specimen, shown here for specimen 2. Cycles go from $15 \mathrm{~N}$ to $120 \mathrm{~N}$. Test was paused after 5 complete cycles, approximately $5 \mathrm{~min}$, and specimen was rotated $180^{\circ}$, indicated by blue arrow, and resumed. Gray markers denote times when three consecutive images were averaged for DIC analysis. The red markers denote the final cycle. The circle markers denote times for reference image averaging, and the square markers denote times for analyzed image averaging. The averaged analyzed image was analyzed using the preceding averaged reference image; each analyzed image had its own reference image. Test ends with tensile failure.

Figure 4: Load-displacement cycles for each specimen; specimen 2 displacement is offset $0.5 \mathrm{~mm}$ for visibility.

Figure 5: Laser printed DIC pattern on specimen, $25 \mathrm{~mm}$ x $25 \mathrm{~mm}$ region.

Figure 6: Magnification of DIC pattern showing $1.8 \mathrm{~mm}$ x $1.8 \mathrm{~mm}$ portion of specimen.

Figure 7: Results from the parametric DIC analysis used to determine optimal DIC regularization parameters as subset size, step, shape function and type of displacement smoothing to derive strains. Yellow marker denotes the strain resolution and VSG used for this work and whose DIC parameters are listed Table 1. Affine and quadratic refer to the adopted shape functions in the correlation algorithms. Q4 and Q8 refer to bilinear and quadratic quadrilateral, respectively, strain interpolation functions.

Figure 8: FEM model used for each simulation. VFM analysis was performed in the necked down portion.

Figure 9: Isotropic specific stiffness, $Q / \rho$, for 1st FEM simulation; $v=0.3$ throughout model.

Figure 10: Isotropic specific stiffness, $Q / \rho$, for 2nd simulation.

Figure 11: Isotropic specific stiffness, $v \cdot Q / \rho$, for 2nd FEM simulation

Figure 12: Strains for 1st FEM simulation.

Figure 13: Identification results for 1st simulation for a different number of Fourier terms. Rbf smoothing factor $=15$.

Figure 14: Analysis of identification for 1st simulation. EG analyses share same scale. Smoothed $Q / \rho$ was used for error and EG analysis.

Figure 15: Strains for 2nd FEM simulation.

Figure 16: Identification results for 2nd simulation for a different number of Fourier terms.

Figure 17: Analysis of identification for 2nd simulation. EG analyses share same scale. 
Figure 18: Normalized $\varepsilon_{\mathrm{y}}$ for each cycle. Specimen is rotated $180^{\circ}$ between 5 th and 6 th cycles as indicated by the arrows. The normalized $\varepsilon_{\mathrm{y}}$ contours are rotated for easier visualization.

Figure 19: $\operatorname{Mean}\left(\varepsilon_{\mathrm{y}}\right)$ and $\operatorname{COV}\left(\varepsilon_{\mathrm{y}}\right)$ for each cycle. Specimen is rotated $180^{\circ}$ between 5 th and 6 th cycles as indicated by the arrows.

Figure 20: Strains for analyses, cycle 11. Nominal size of area shown is $41 \mathrm{~mm}$ x $124 \mathrm{~mm}$.

Figure 21: Homogeneity of $\varepsilon_{\mathrm{y}}$ for PET specimen as compared to the paperboard specimens.

Figure 22: Von Mises equivalent strain histograms for each specimen. Divisions for the lowest standard deviation of EG are indicated.

Figure 23: Material divisions based on Von Mises equivalent strain for each specimen.

Figure 24: Strains and normalized stiffnesses for each paperboard specimen. Rbf smoothing $=15$. Horizontal gray lines represent median vertical failure location. 
Table 1: DIC system components and parameters

\begin{tabular}{ll}
\hline Technique & Stereo DIC \\
Camera & Allied Vision Technologies \\
& (Stadtroda, Germany) \\
& Stingray Model F504B \\
& Computar (Commack, NY) \\
Lens & M1614-MP2, 16 mm, \\
& f1.4 \\
& $0.373 \mathrm{~m}$ \\
Imaging distance & White fluorescent \\
Lighting & $2452 \times 2056,12-$ bit \\
Resolution & $0.4 \%$ of dynamic range \\
Noise & MatchID v2016.1.1 \\
Software & $0.5 \mathrm{~Hz}$ \\
Capture rate & 1 pixel $=0.036 \mathrm{~mm}$ \\
Pixel-mm conversion & $150 \mathrm{~mm} \times 50 \mathrm{~mm}$ \\
ROI & 21,10 (pixels) \\
Subset, step & Cubic spline, affine, \\
Interpolation, shape & ZNSSD \\
functions, & \\
correlation criterion & \\
Pre-smoothing & Gaussian 5 \\
Displacement, in- & 0.021 pixels, 0.39 $\mu \mathrm{m}$ \\
plane resolution & \\
Strain & \\
Calculation & Improved quadratic \\
& quadrilateral \\
Strain window & 9 data points \\
VSG (virtual strain & 101 pixels, 3.64 mm \\
gage) & \\
In-plane resolution & $65 \mu \mathrm{m} / \mathrm{m}$ \\
\hline &
\end{tabular}


1

2

3

4

5

6

7

8

9

10

11

12

13

14

15

16

17

18

19

20

21

22

23

24

25

26

27

28

29

30

31

32

33

34

35

36

37

38

39

40

41

42

43

44

45

46

47

48

49

50

51

52

53

54

55

56

57

58

59

60

Table 2: Results for both simulations ${ }^{\mathrm{a}}$

\begin{tabular}{lcrrr}
\hline & $\begin{array}{c}\text { Percentile } \\
\text { (unless } \\
\text { noted) }\end{array}$ & & & \\
& Homogeneous & Heterogeneous & $\begin{array}{r}\text { Rbf smoothed } \\
\text { heterogeneous }\end{array}$ \\
\hline Simulation 1 & & & & \\
\% error & 90th & 1.17 & 13.14 & 12.18 \\
& 50th & 1.17 & 8.18 & 7.80 \\
& 10th & -0.56 & -8.48 & -3.83 \\
EG analysis & std(error) & 3.27 & 10.27 & 7.32 \\
& 90th & 22.73 & 318.06 & 267.20 \\
& 50th & 0.58 & -3.45 & -4.02 \\
Simulation 2 & 10th & -22.83 & -335.99 & -285.39 \\
\% error & std(EG) & 295.61 & 341.52 & 302.99 \\
& 90th & -2.18 & 13.15 & 12.76 \\
& 50th & -2.33 & 7.99 & 7.53 \\
& 10th & -3.41 & -9.61 & -5.17 \\
EG analysis & std(error) & 3.06 & 9.83 & 7.84 \\
& 90th & 122.55 & 584.13 & 501.58 \\
& 50th & & -1.73 & -0.57 \\
& 10th & -206.70 & -741.31 & -673.17 \\
& std(EG) & 386.82 & 742.13 & 681.23 \\
\hline
\end{tabular}

${ }^{\mathrm{a}}$ Heterogeneous results represent $N=12$ in Equation (5). EG has units specific stiffness/area $\left(\mathrm{s}^{-2} 10^{17}\right)$. Smoothing factor for $\mathrm{Rbf}=15$. 
Table 3: Results of VFM analyses for the tensile specimens ${ }^{\mathrm{a}}$

\begin{tabular}{|c|c|c|c|c|c|c|c|c|c|c|}
\hline \multirow[b]{2}{*}{ Specimen } & \multirow[b]{2}{*}{ Analysis } & \multicolumn{3}{|c|}{$Q$ percentiles } & \multicolumn{4}{|c|}{ EG percentiles } & \multirow[b]{2}{*}{ Voight } & \multirow[b]{2}{*}{ Reuss } \\
\hline & & 75.0 & 32.5 & 7.5 & 90 & 50 & 10 & Std & & \\
\hline \multirow[t]{14}{*}{1} & Homogeneous & & & & & & & & & \\
\hline & $\operatorname{mean}\left(\varepsilon_{y}\right) Q$ & & 3.46 & & & & & & & \\
\hline & $v$ & & 0.17 & & & & & & & \\
\hline & $\operatorname{VFM} Q$ & & 3.60 & & 110.3 & -25.5 & -100.5 & 87.6 & & \\
\hline & & & 0.18 & & & & & & & \\
\hline & Segmented; divisi & is: $85^{\circ}$ & $50 \%$ o & & & & & & & \\
\hline & $Q$ & 4.63 & 3.86 & 3.46 & 73.8 & -15.3 & -83.8 & 63.3 & 4.18 & 4.13 \\
\hline & $v$ & 0.21 & 0.15 & 0.17 & & & & & & \\
\hline & F-VFM; $N=7$ & & & & & & & & & \\
\hline & $Q$ & 3.93 & 3.21 & 2.73 & 83.7 & -6.9 & -90.3 & 65.7 & 3.57 & 3.46 \\
\hline & Smoothed F-VFM & $N=7$ & of smo & thing = & & & & & & \\
\hline & $Q$ & 3.91 & 3.20 & 2.78 & 75.5 & -7.5 & -84.5 & 60.7 & 3.57 & 3.47 \\
\hline & & & ercenti & & & EG per & entiles & & & \\
\hline & & 70.0 & 30.0 & 10.0 & 90 & 50 & 10 & Std & Voight & Reuss \\
\hline \multirow[t]{12}{*}{2} & Homogeneous & & & & & & & & & \\
\hline & $\operatorname{mean}\left(\varepsilon_{y}\right) Q$ & & 3.98 & & & & & & & \\
\hline & $v$ & & 0.12 & & & & & & & \\
\hline & VFM $Q$ & & 4.11 & & 72.9 & -16.9 & -104.2 & 66.8 & & \\
\hline & $v$ & & 0.19 & & & & & & & \\
\hline & Segmented; divisi & Is: $60^{\circ}$ & $80 \%$ o & & & & & & & \\
\hline & $Q$ & 4.61 & 4.00 & 3.72 & 63.7 & -16.2 & -74.7 & 55.2 & 4.31 & 4.28 \\
\hline & $v$ & 0.16 & 0.20 & 0.22 & & & & & & \\
\hline & F-VFM; $N=7$ & & & & & & & & & \\
\hline & $Q$ & 3.99 & 3.48 & 3.20 & 58.6 & -6.2 & -83.7 & 53.7 & 3.81 & 3.74 \\
\hline & Smoothed F-VFM & $N=7$, & of smo & thing $=$ & & & & & & \\
\hline & $Q$ & 3.99 & 3.53 & 3.24 & 53.8 & -6.7 & -77.1 & 49.8 & 3.81 & 3.76 \\
\hline
\end{tabular}




\section{Page 31 of 54}

Strain

1

2

3

4

5

6
7

8

9

10

Figure 1: Schematic of Equilibrium Gap mesh, which was rastered throughout inspection region. 


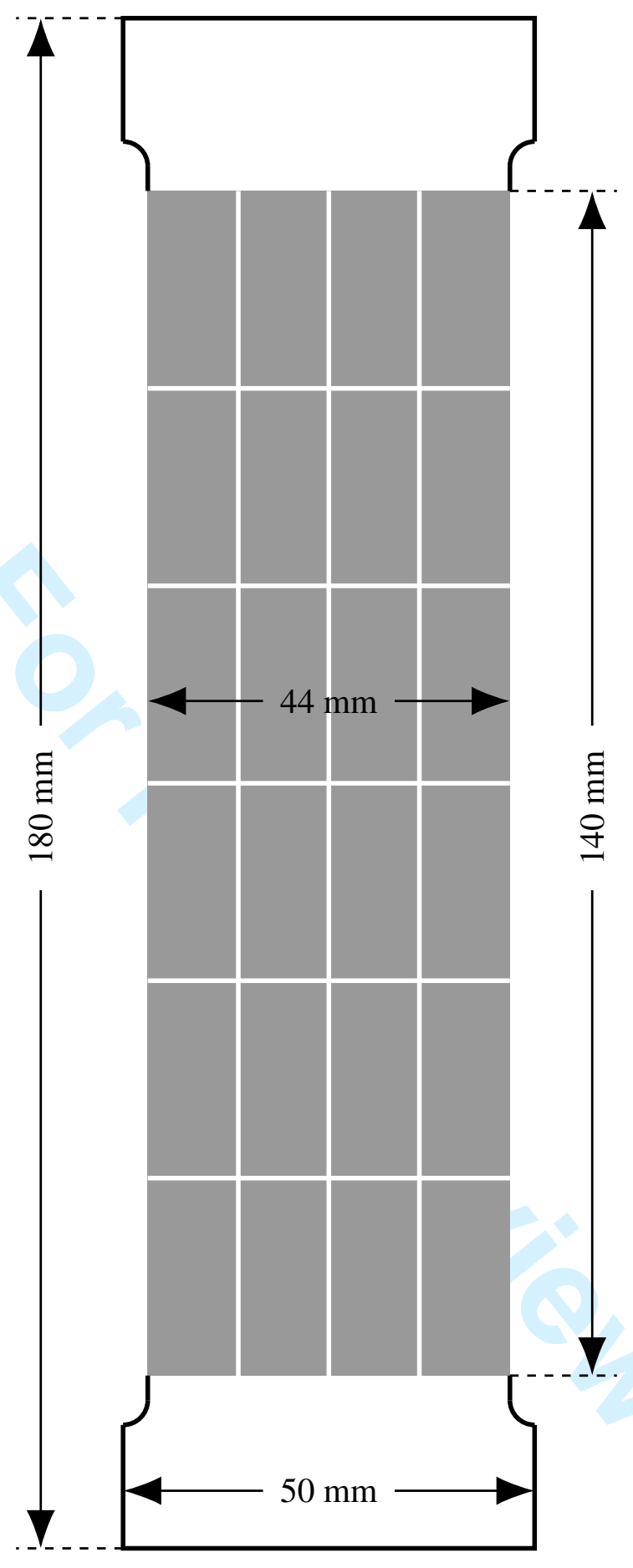

Figure 2: Paperboard specimen geometry, approximately to scale. Area inspected by VFM is filled in gray. White lines within inspection region represent VFM mesh for homogeneous and segmented heterogeneous identification. 


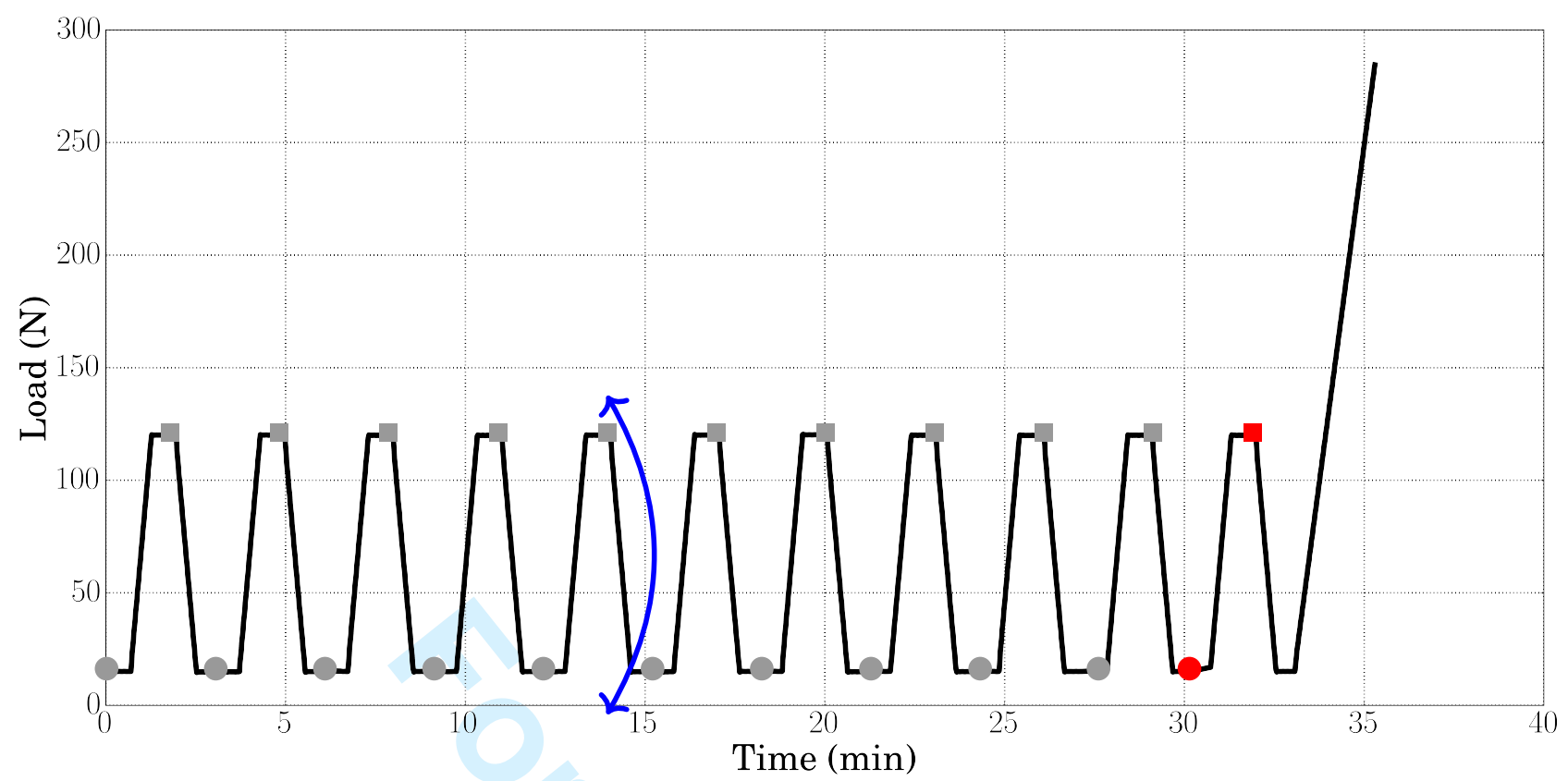

Figure 3: Load sequence for each specimen, shown here for specimen 2. Cycles go from $15 \mathrm{~N}$ to 120 $\mathrm{N}$. Test was paused after 5 complete cycles, approximately 5 minutes, and specimen was rotated $180^{\circ}$, indicated by blue arrow, and resumed. Gray markers denote times when three consecutive images were averaged for DIC analysis. The red markers denote the final cycle. The circle markers denote times for reference image averaging, and the square markers denote times for analyzed image averaging. The averaged analyzed image was analyzed using the preceding averaged reference image; each analyzed image had its own reference image. Test ends with tensile failure. 
1

2

3

4

5

6

7

8

9

10

11

12

13

14

15

16

17

18

19

20

21

22

23

24

25

26

27

28

29

30

31

32

33

34

35

36

37

38

39

40

41

42

43

44

45

46

47

48

49

50

51

52

53

54

55

56

57

58

59

60

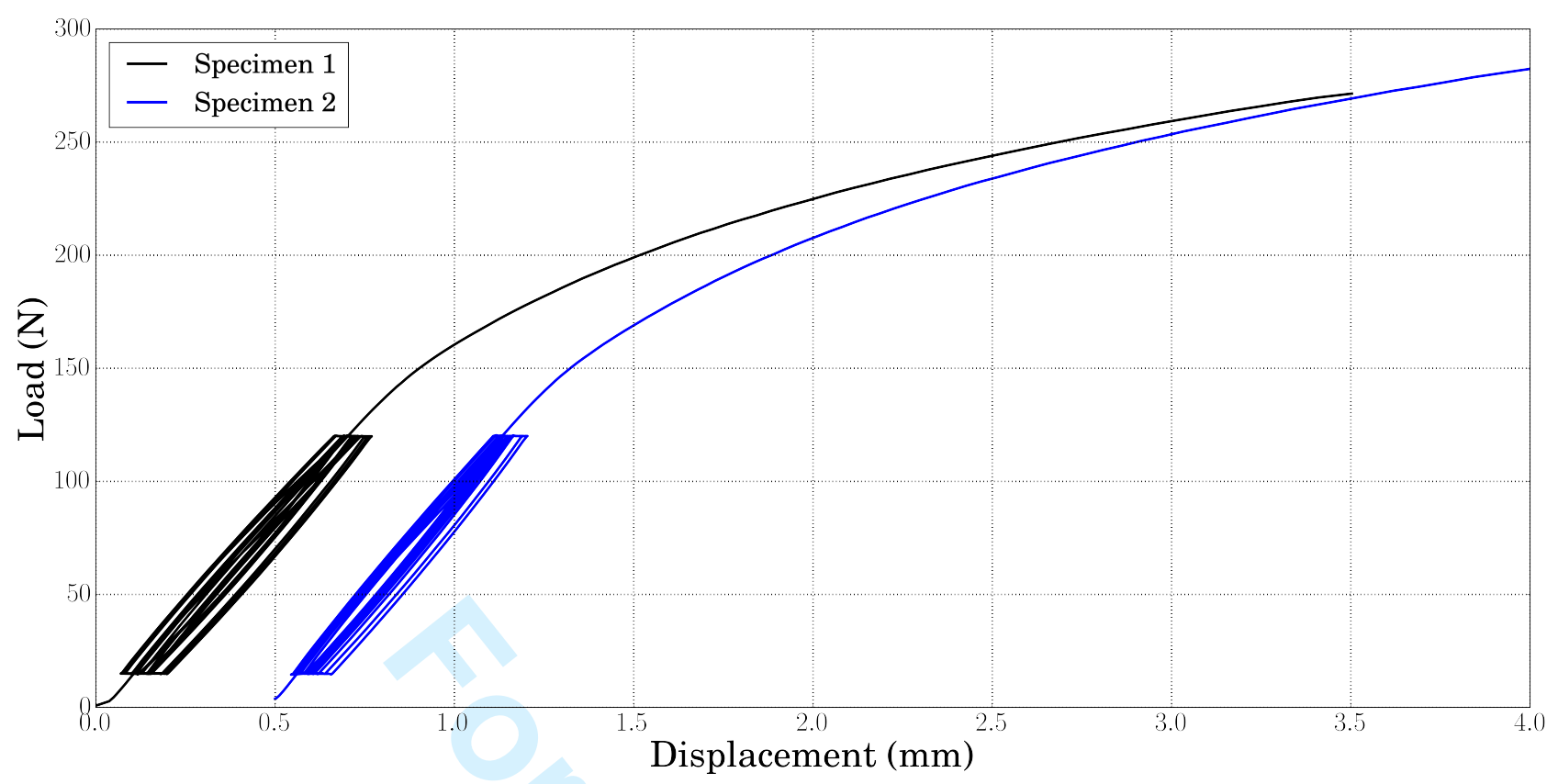

Figure 4: Load-displacement cycles for each specimen; specimen 2 displacement is offset $0.5 \mathrm{~mm}$ for visibility. 


\section{Page 35 of 54}

Strain

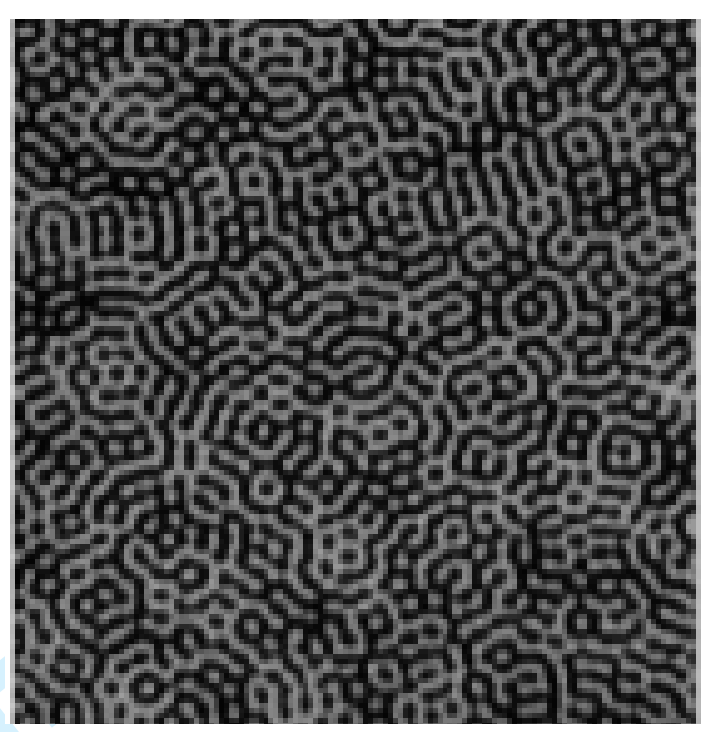

Figure 5: Laser printed DIC pattern on specimen $25 \mathrm{~mm} \times 25 \mathrm{~mm}$ region. 


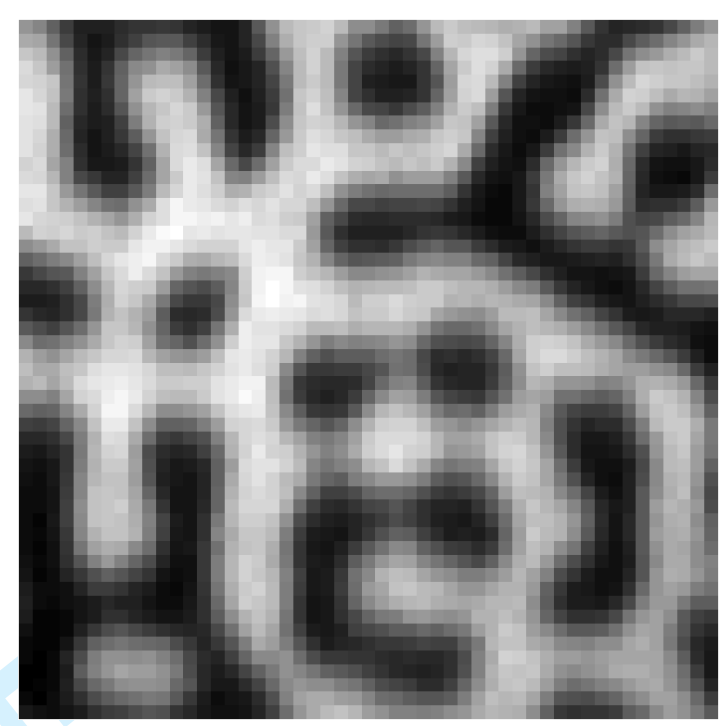

Figure 6: Magnification of DIC pattern showing $1.8 \mathrm{~mm} \times 1.8 \mathrm{~mm}$ portion of specimen. 


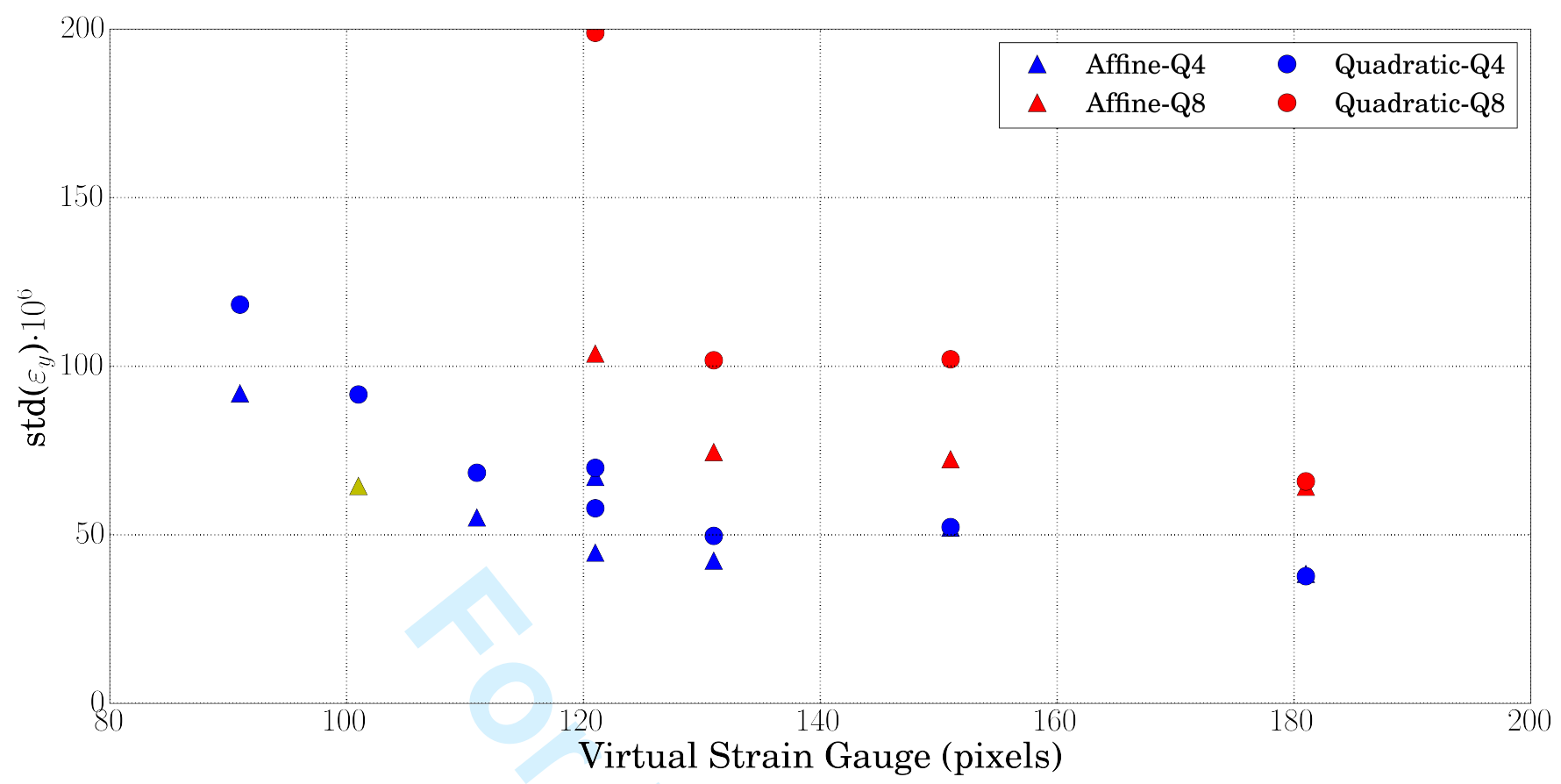

Figure 7: Results from the parametric DIC analysis used to determine optimal DIC regularization parameters as subset size, step, shape function and type of displacement smoothing to derive strains. Yellow marker denotes the strain resolution and VSG used for this work and whose DIC parameters are listed Table 1. Affine and quadratic refer to the adopted shape functions in the correlation algorithms. Q4 and Q8 refer to bilinear and quadratic quadrilateral, respectively, strain interpolation functions . 


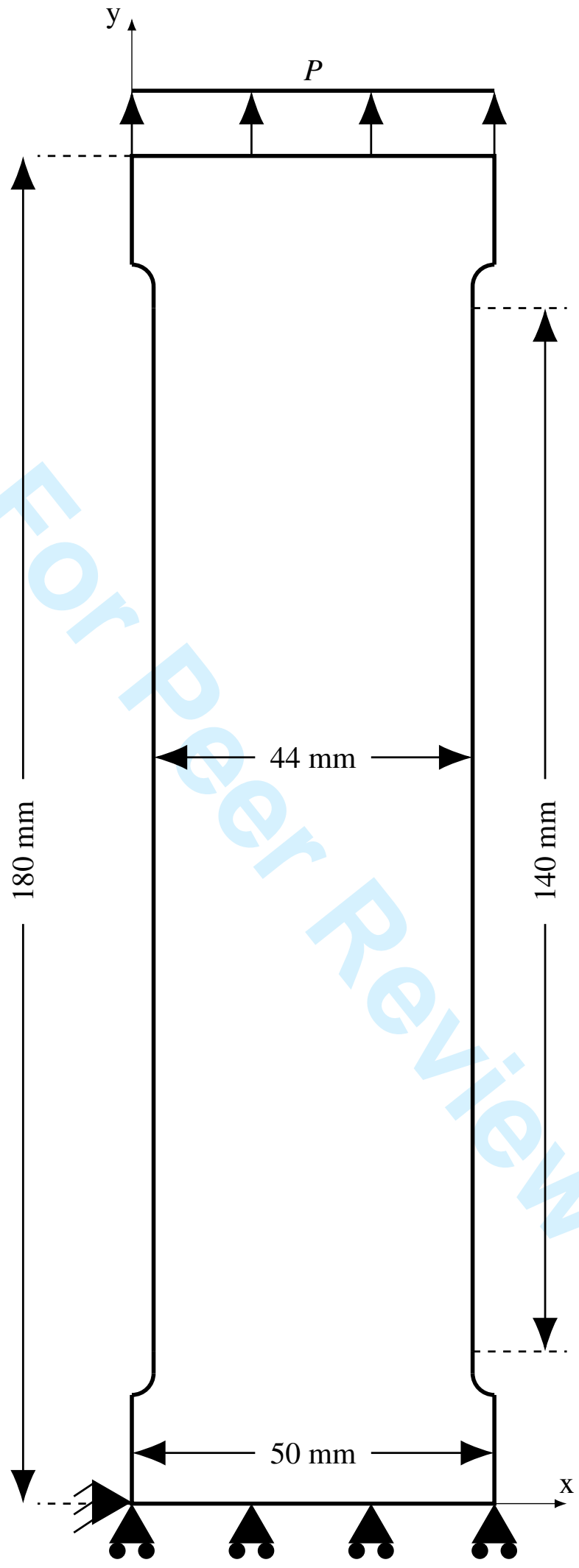

Figure 8: FEM model used for the two simulations. VFM analysis was performed in the necked down portion. 
1

2

3

4

5

6
7

8

9

10

11

12

13

14

15

16

17

18

19

20

21

22

23

24

25

26

27

28

29

30

31

32

33

34

35

36

37

38

39

40

41

42

43

44

45

46

47

48

49

50

51

52

53

54

55

56

57

58

59

60

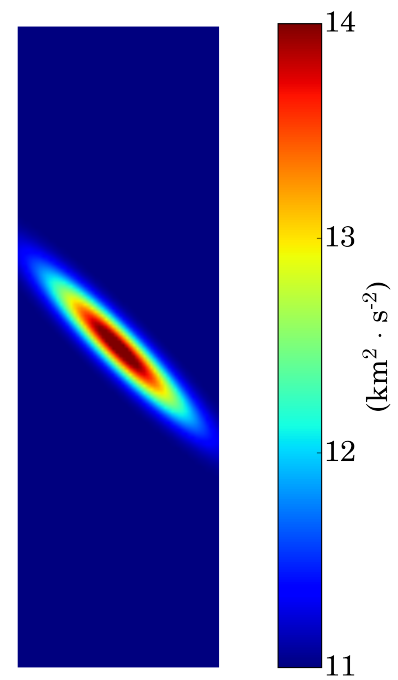

Figure 9: Isotropic specific stiffness, $Q / \rho$, for 1st FEM simulation; $v=0.3$ throughout model. 


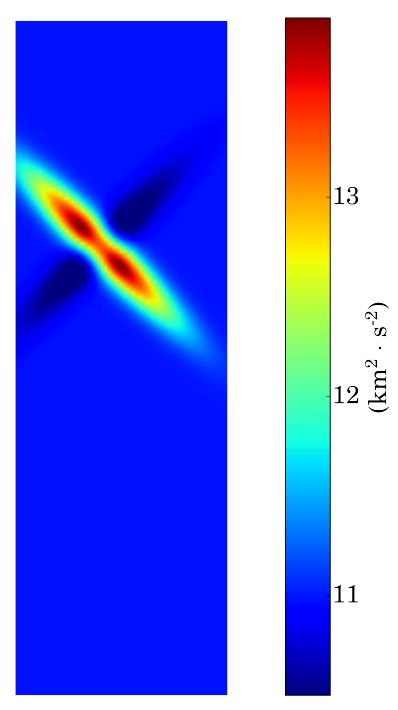

Figure 10: Isotropic specific stiffness, $Q / \rho$, for 2nd FEM simulation. 


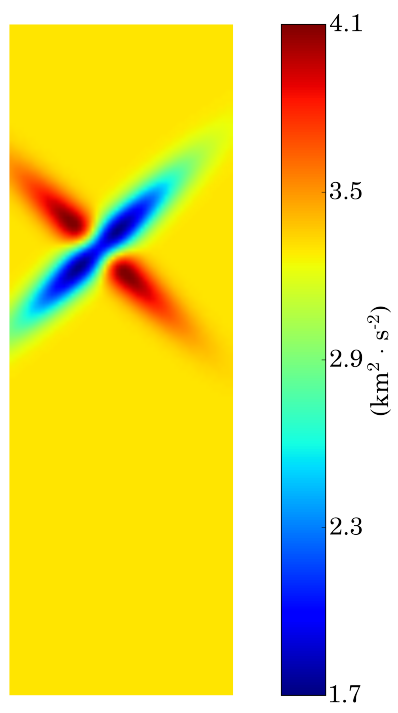

Figure 11: Isotropic specific stiffness, $v \cdot Q / \rho$, for 2nd FEM simulation. 

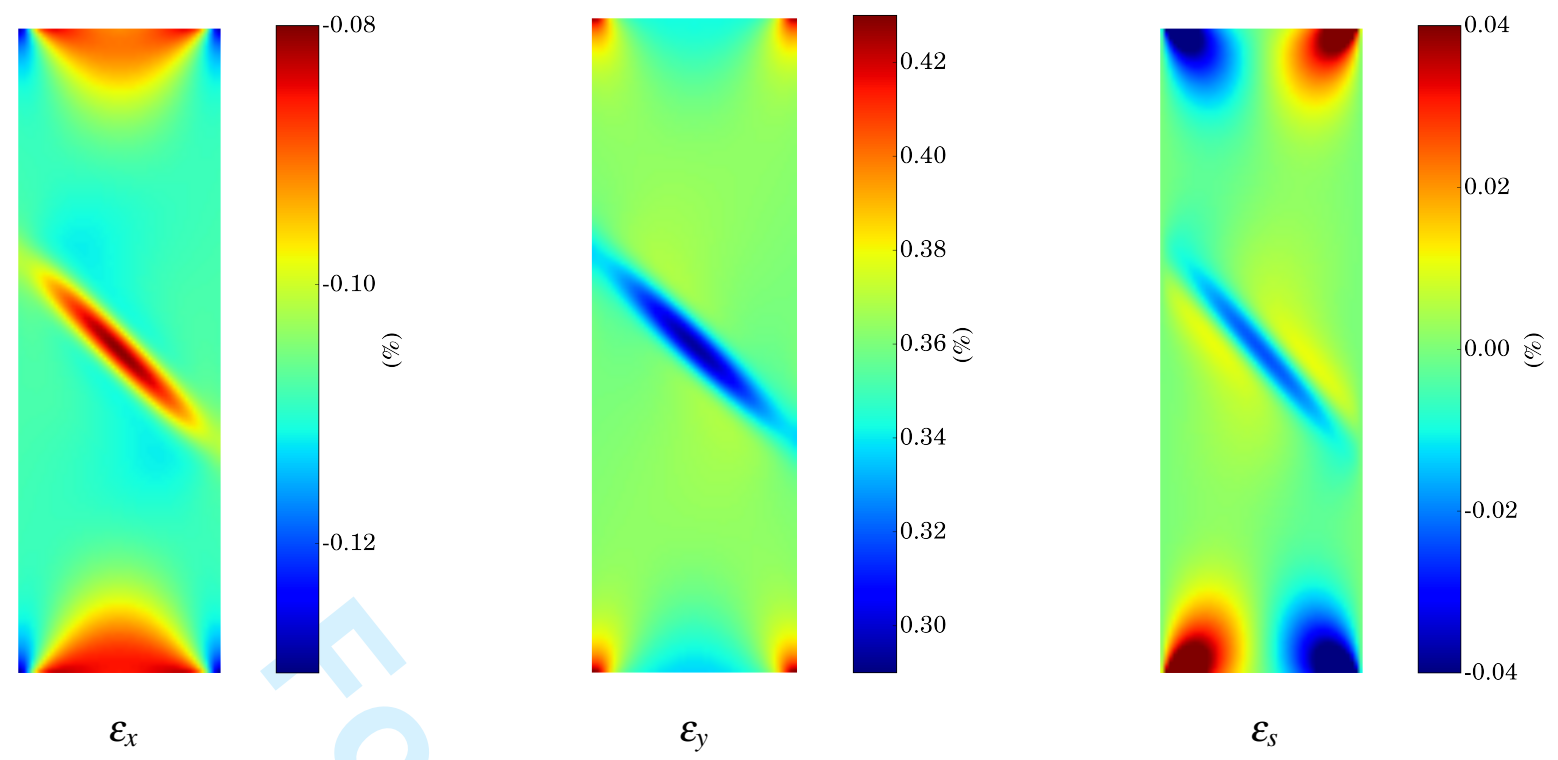

Figure 12: Strains for 1st FEM simulation. 


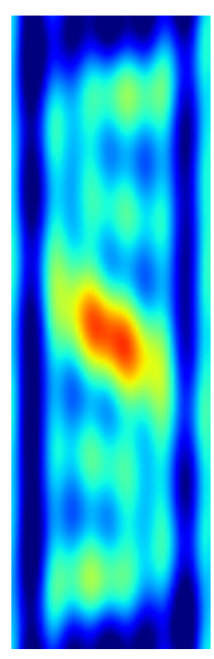

$N=5$

131 terms

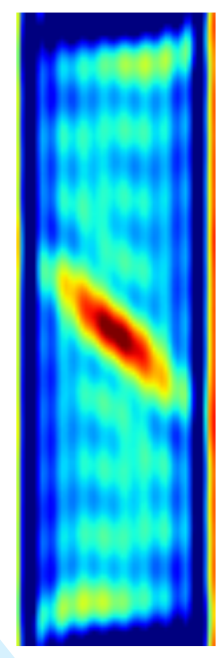

$$
N=9
$$

379 terms

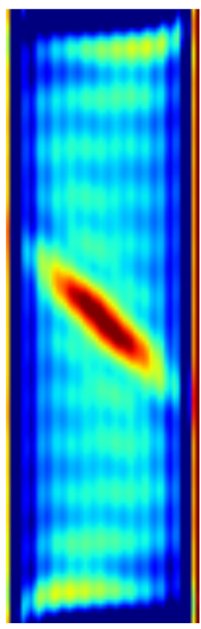

$$
N=12
$$

469 terms

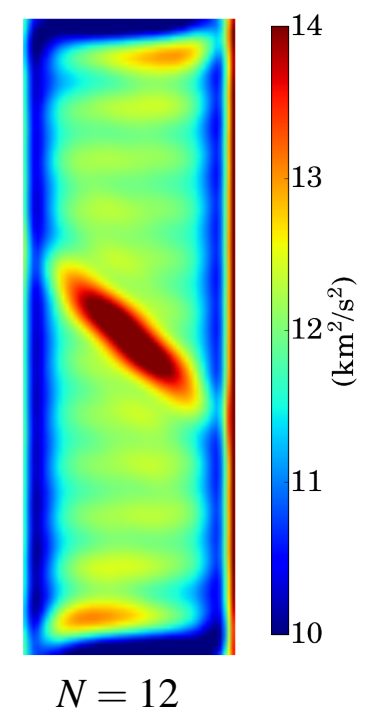

Rbf Smoothed

Figure 13: Identification results for 1st simulation for a different number of Fourier terms. Rbf smoothing factor $=15$. 


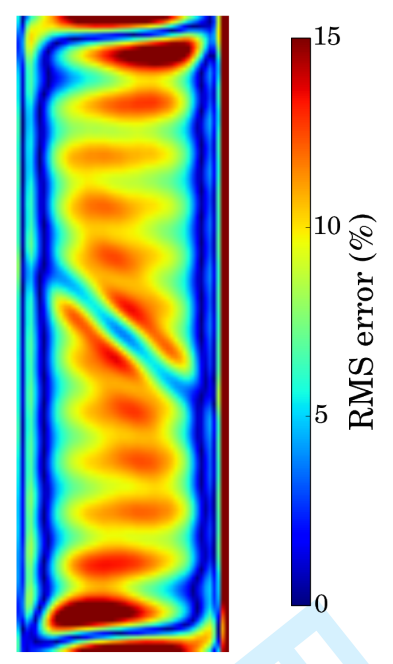

Error for $N=12$ identification

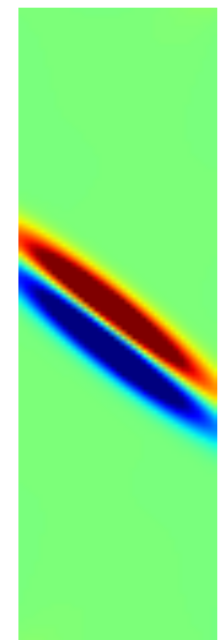

Homogeneous EG

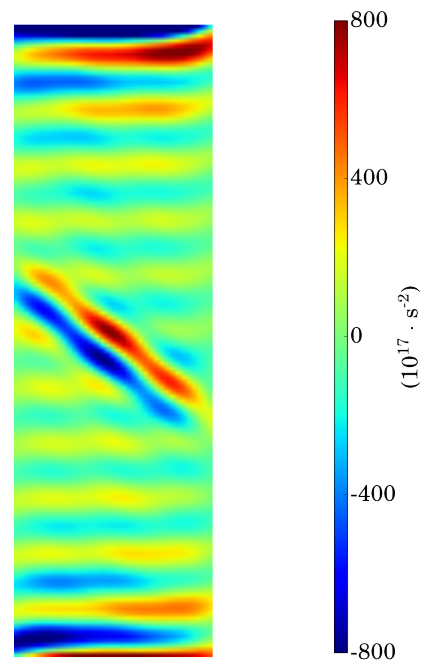

$N=12$, Heterogeneous EG

Figure 14: Analysis of identification for 1st simulation. EG analyses share same scale. Smoothed $Q / \rho$ was used for error and EG analysis. 


\section{Page 45 of 54}

Strain
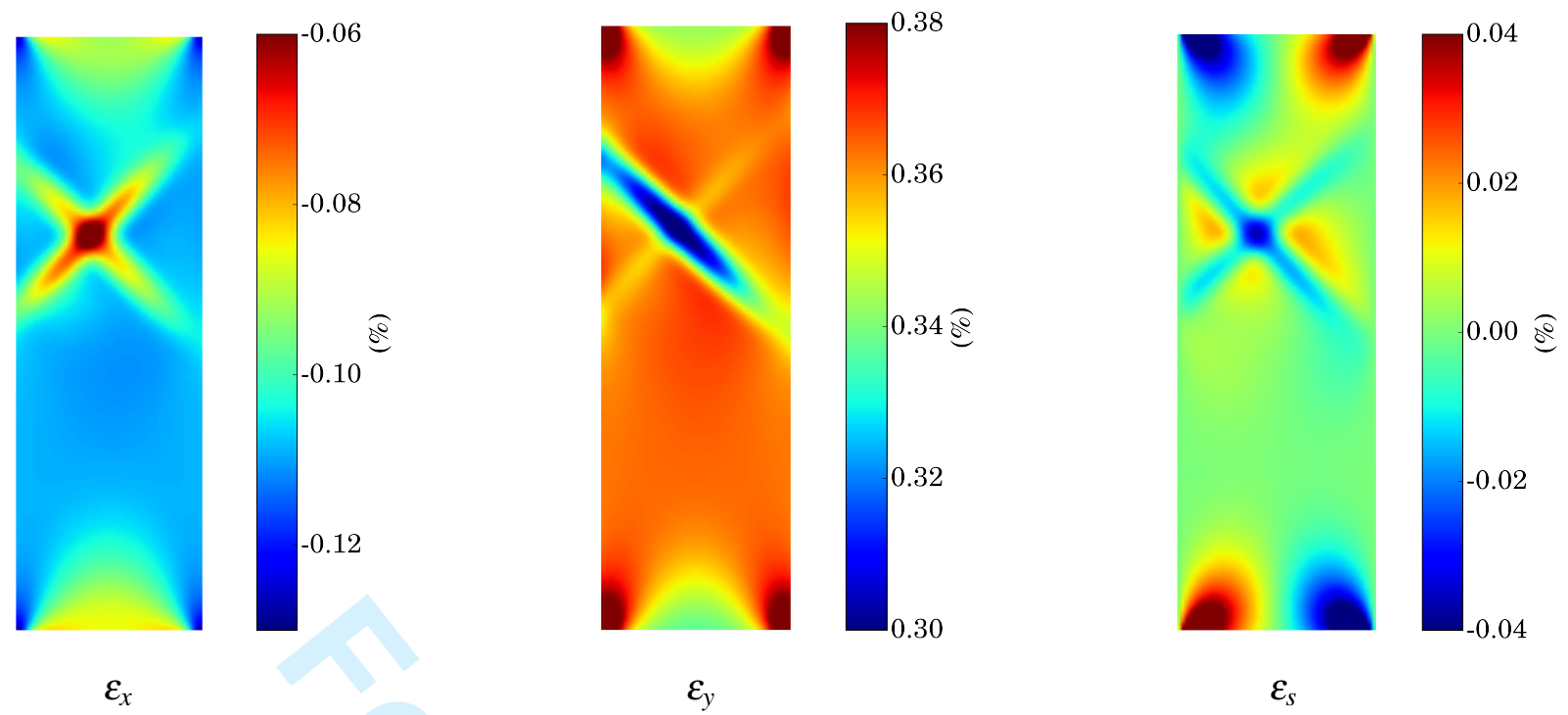

Figure 15: Strains for 2nd FEM simulation.

1
2
3
4
5
6
7
8
9
10
11
12
13
14
15
16
17
18
19
20
21
22
23
24
25
26
27
28
29
30
31
32
33
34
35
36
37
38
39
40
59
50
50
48
49
50
51
52
53
54

1

2

4

5
6

7

9

10

12

13

14
15

16

17

19

20

21

23

24

25

26

27

28

29

31

32

33

34

35

36

37

38

39

40

41

42

43

44

46

47

48

49

50

51

53

54

55

56

57

58

60 


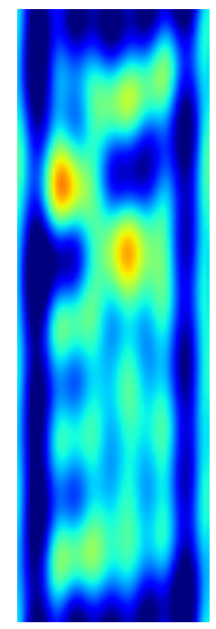

$N=5$

131 terms

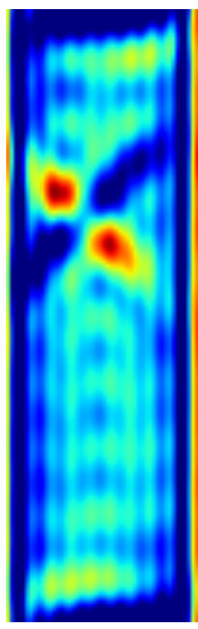

$$
N=9
$$

379 terms

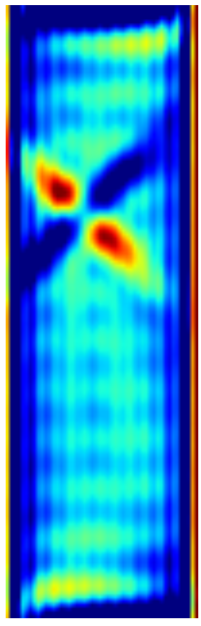

$N=12$

469 terms

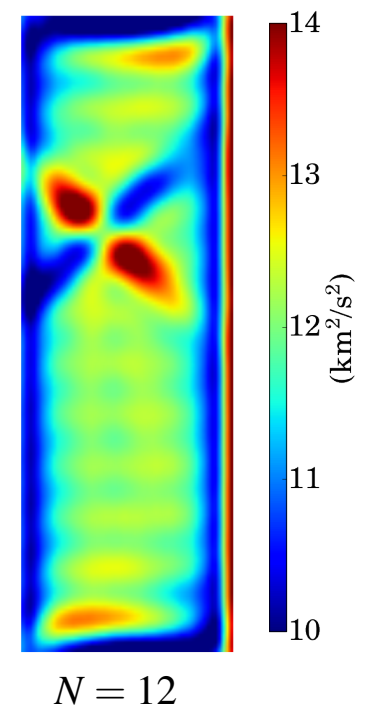

Rbf Smoothed

Figure 16: Identification results for 2nd simulation for a different number of Fourier terms. Rbf smoothing factor $=15$. 


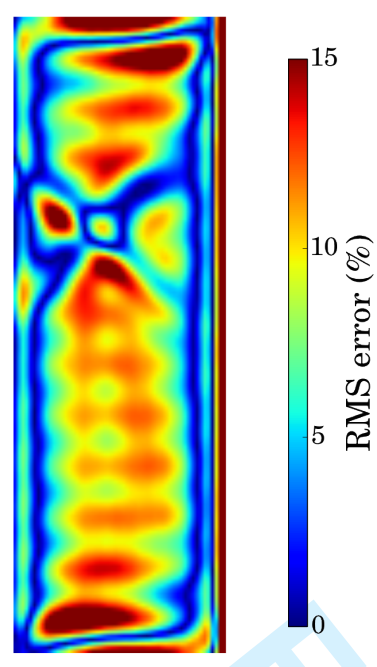

Error for $N=12$ identification
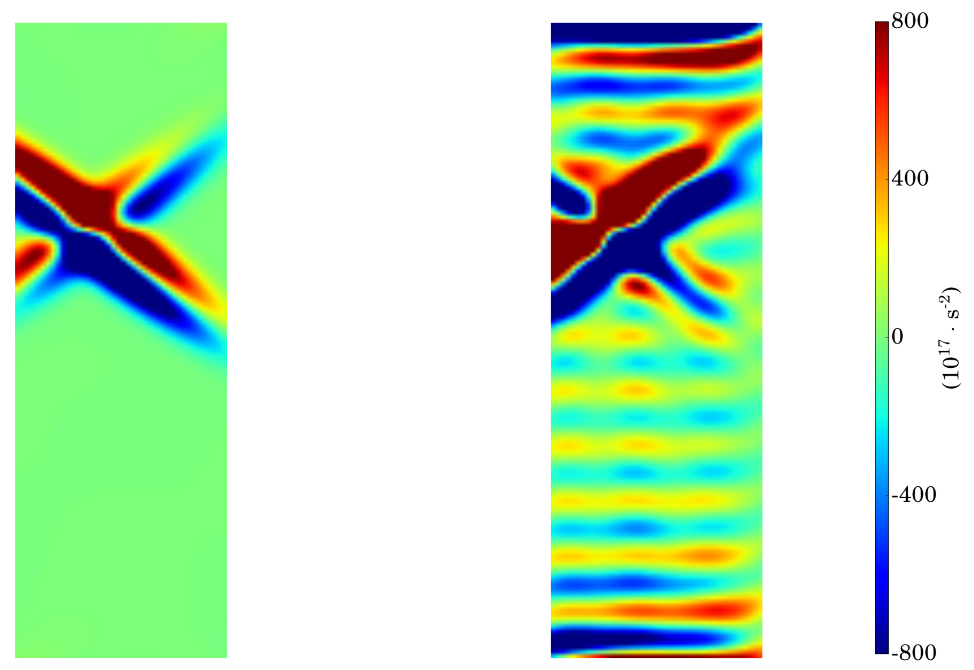

Homogeneous EG $N=12$, Heterogeneous EG

Figure 17: Analysis of identification for 2nd simulation. EG analyses share same scale. Smoothed $Q / \rho$ was used for error and EG analysis. 
Strain

1

2

3

4

5

6

7

8

9

10

11

12

13

14

15

16

17

30

31

32

33

34

35

36

37

38

39

40

41

42

43

44

45

46

47

48

49

50

51

52

53

54

55

56

57

58

59

60

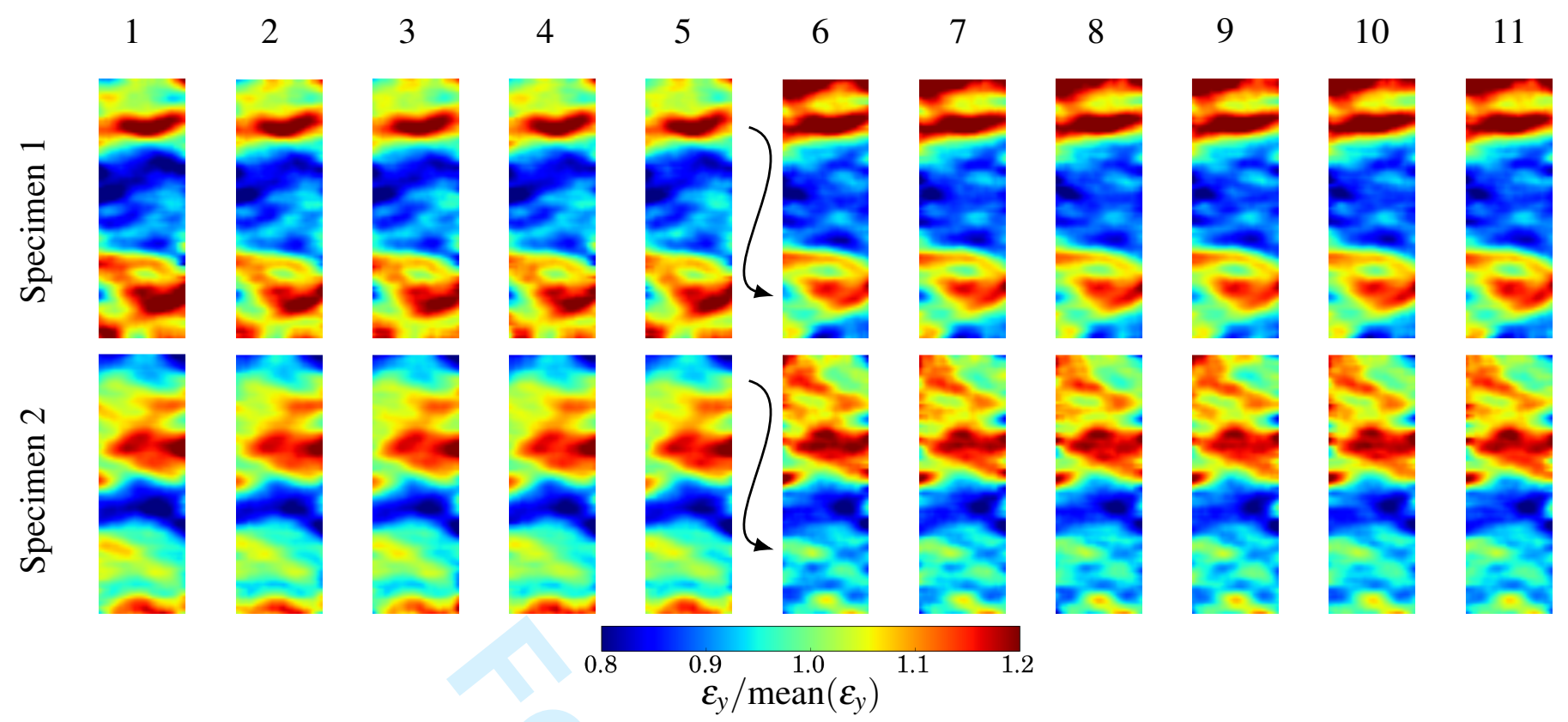

Figure 18: Normalized $\varepsilon_{y}$ for each cycle. Specimen is rotated $180^{\circ}$ between 5 th and 6 th cycles as indicated by the arrows. The normalized $\varepsilon_{y}$ contours are rotated for easier visualization. 


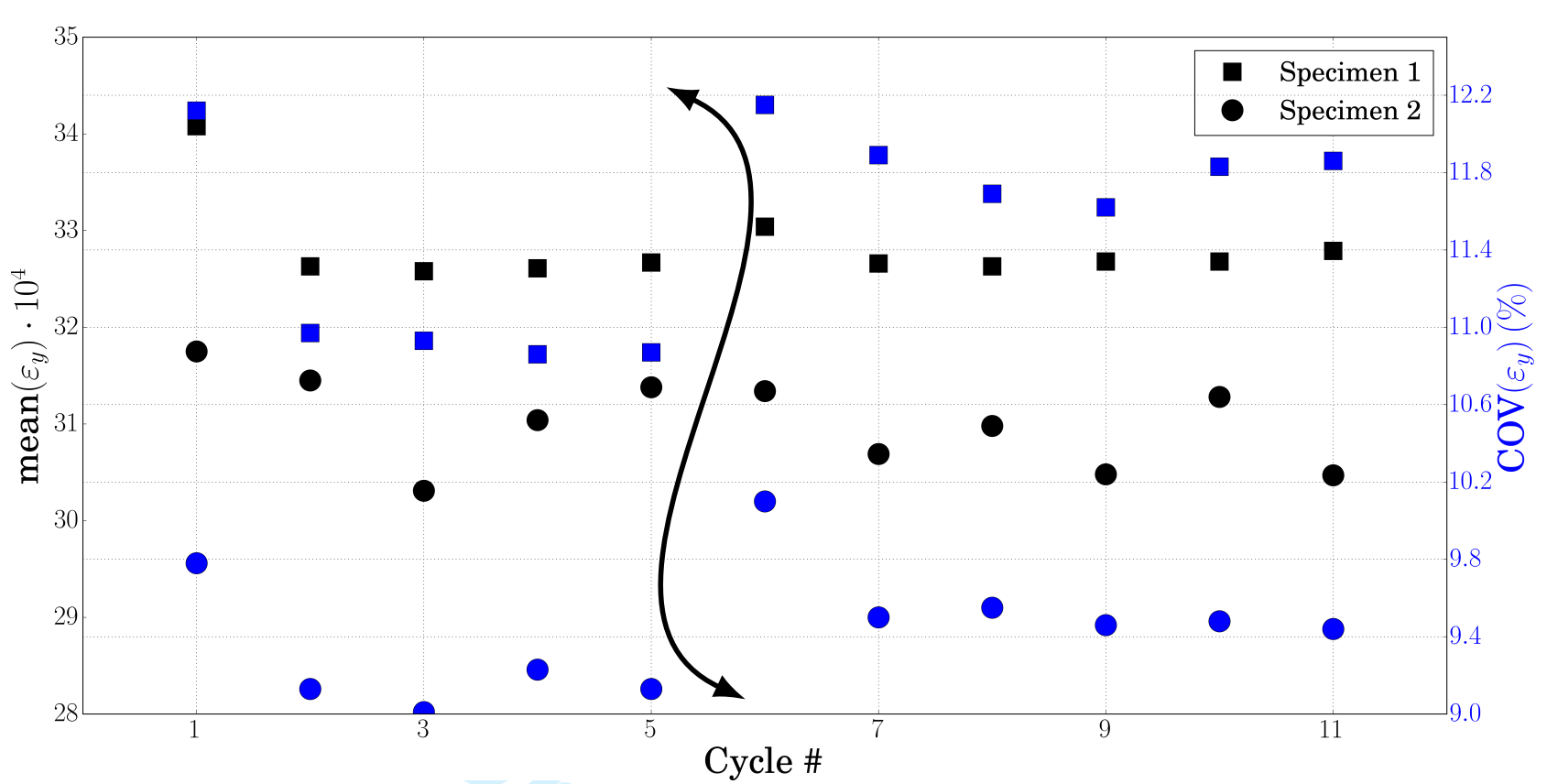

Figure 19: $\operatorname{Mean}\left(\varepsilon_{y}\right)$ and $\operatorname{COV}\left(\varepsilon_{y}\right)$ for each cycle. Specimen is rotated $180^{\circ}$ between 5 th and 6 th cycles as indicated by the arrows. 

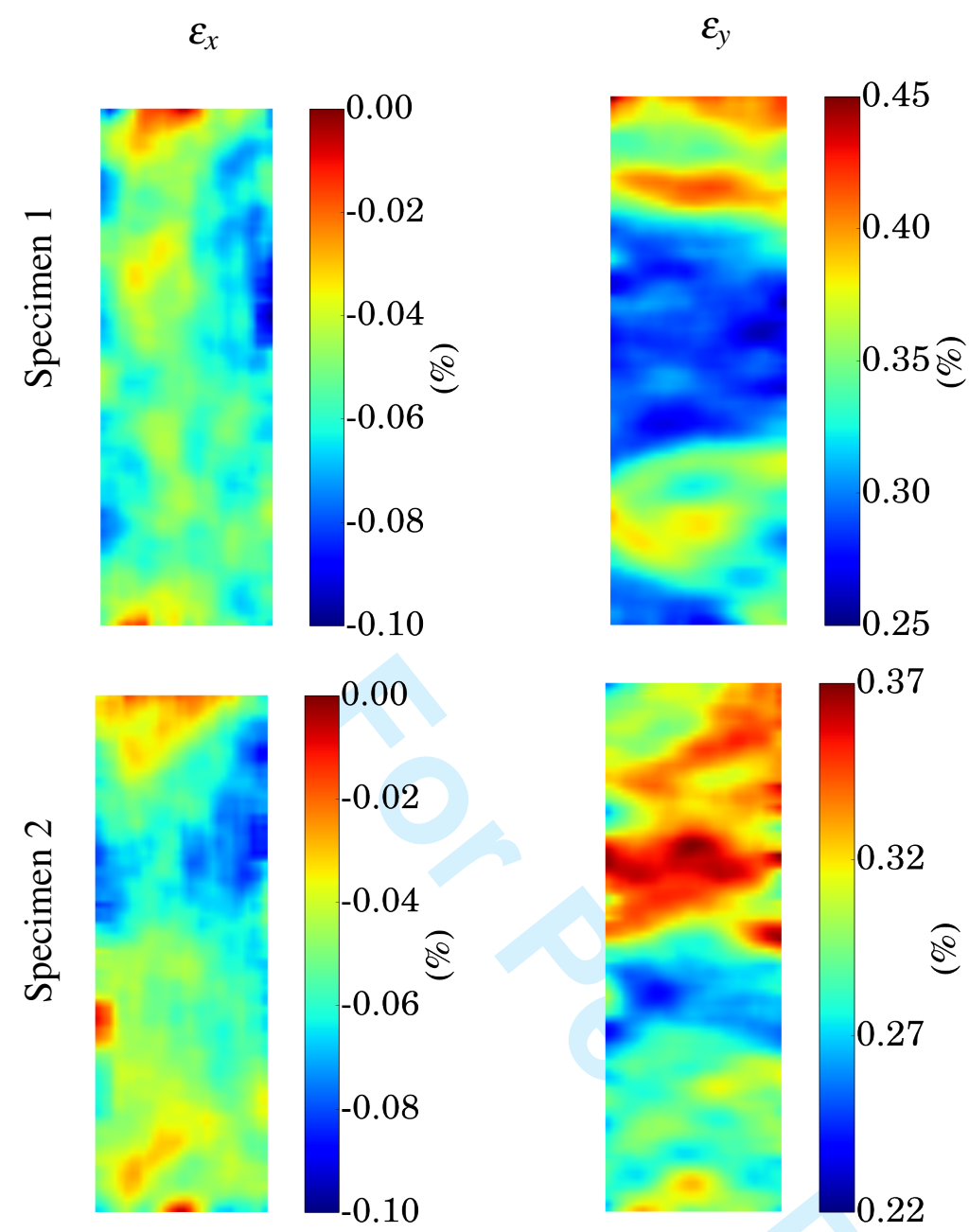

$\varepsilon_{S}$

Figure 20: Strains for analyses, cycle 11 . Nominal size of area shown is $41 \mathrm{~mm} \times 124 \mathrm{~mm}$ 
1

2

3

4

5

6

7

8

9

10

11

12

13

14

15

16

17

18

19

20

21

22

23

24

25

26

27

28

29

30

31

32

33

34

35

36

37

38

39

40

41

42

43

44

45

46

47

48

49

50

51

52

53

54

55

56

57

58

59

60

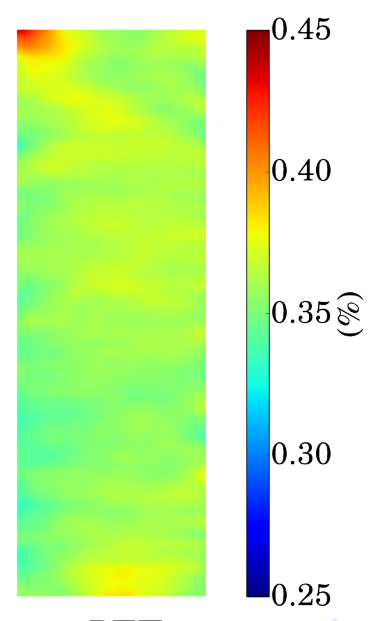

PET

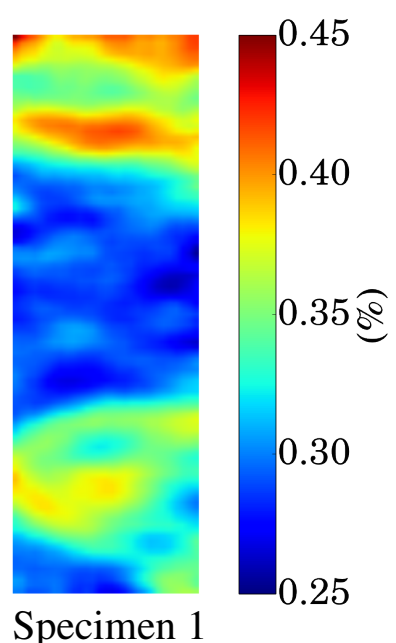

Specimen 1

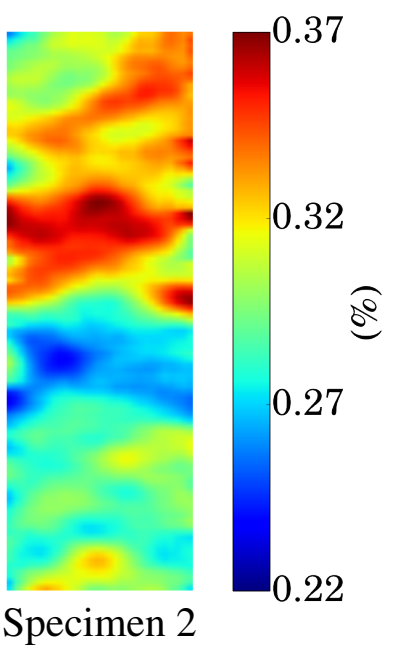

Figure 21: Homogeneity of $\varepsilon_{y}$ for PET specimen as compared to the paperboard specimens. 
1

2

3

4

5

6

7

8

9

10

11

12

13

14

15

16

17

18

19

20

21

22

23

24

25

26

27

28

29

30

31

32

33

34

35

36

37

38

39

40

41

42

43

44

45

46

47

48

49

50

51

52

53

54

55

56

57

58

59

60
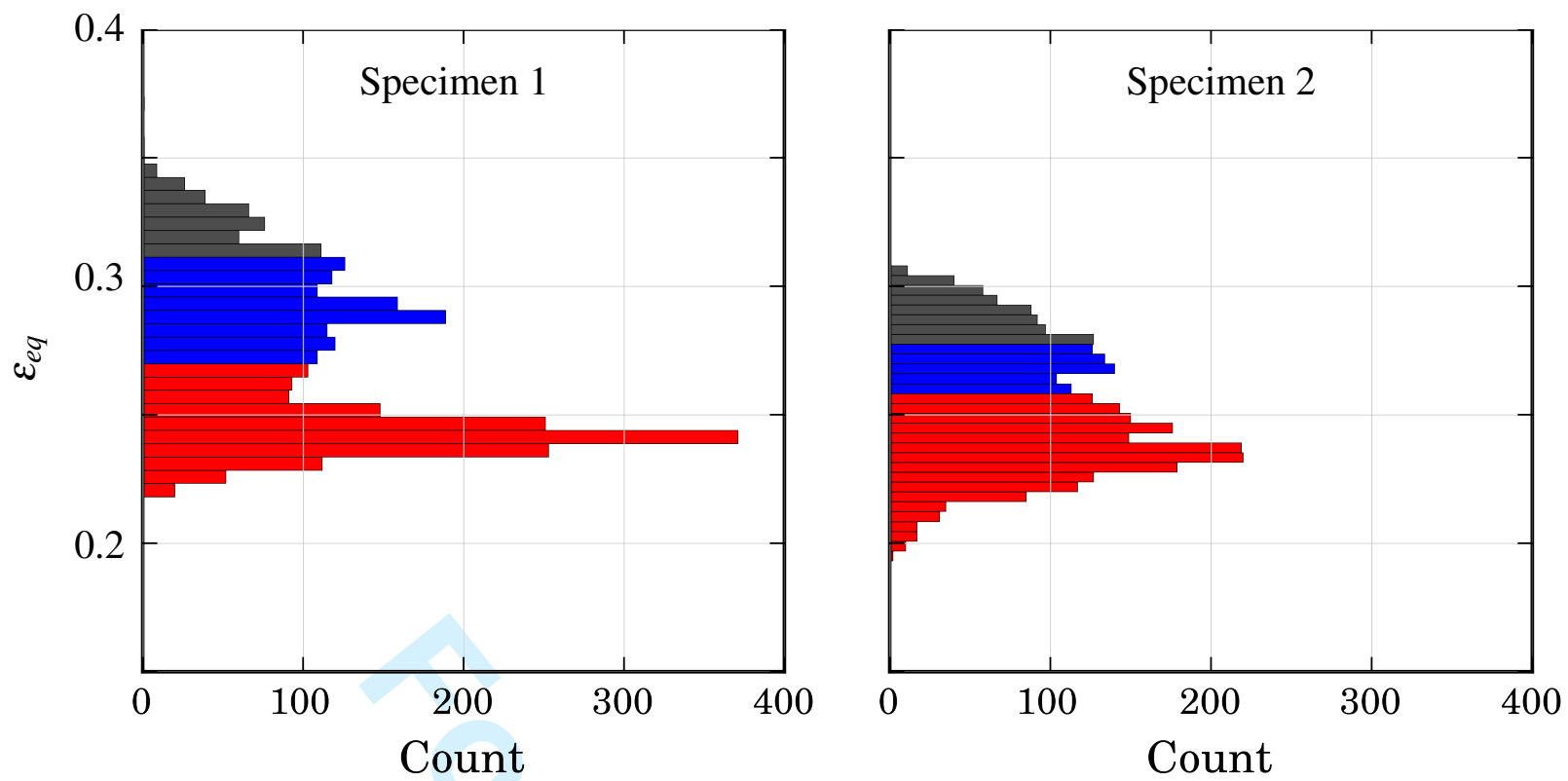

Figure 22: Von Mises equivalent strain histograms for each specimen. Divisions for the lowest standard deviation of EG are indicated. 
Specimen 1
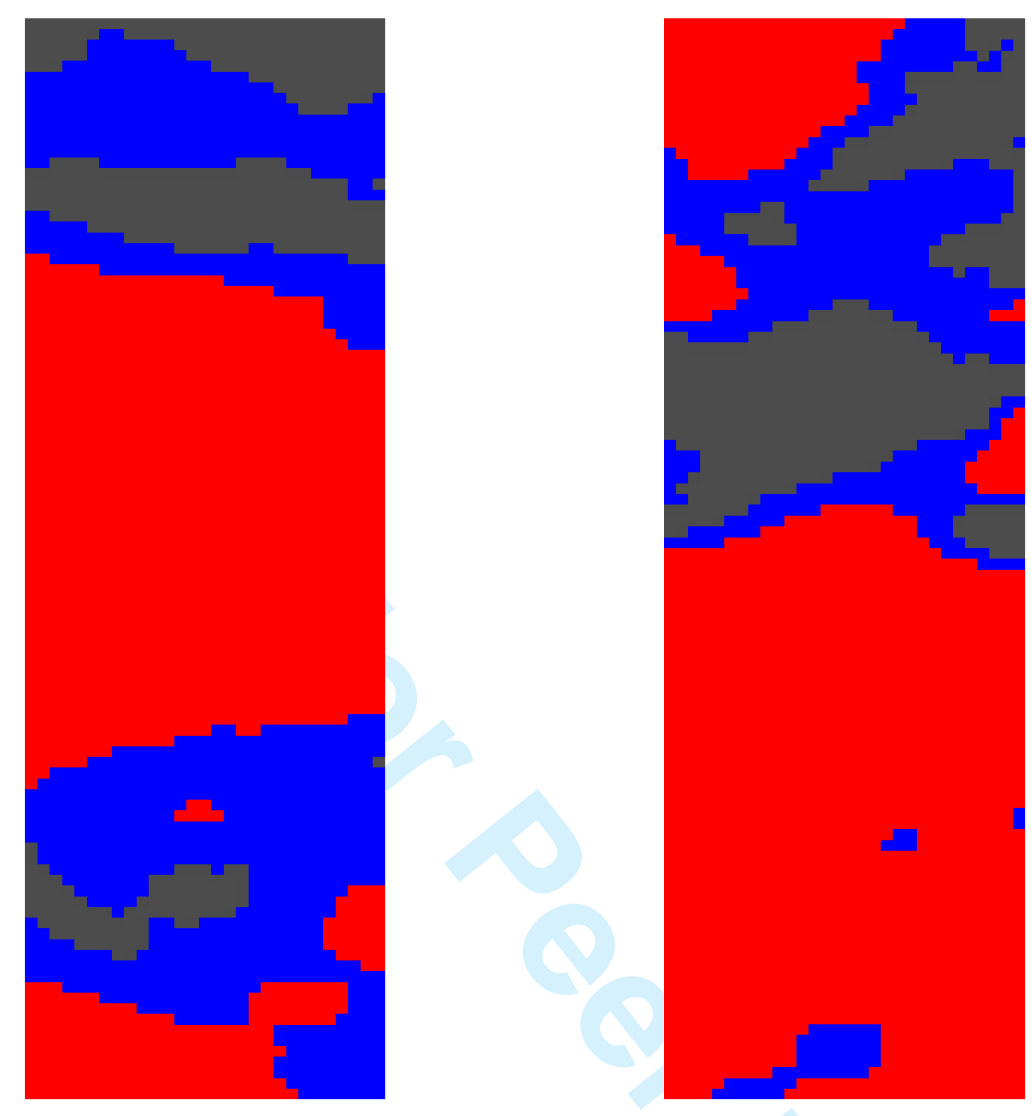

hi $\varepsilon_{e q}$

$\operatorname{med} \varepsilon_{e q}$

lo $\varepsilon_{e q}$

Figure 23: Material divisions based on Von Mises equivalent strain for each specimen. 
1

2
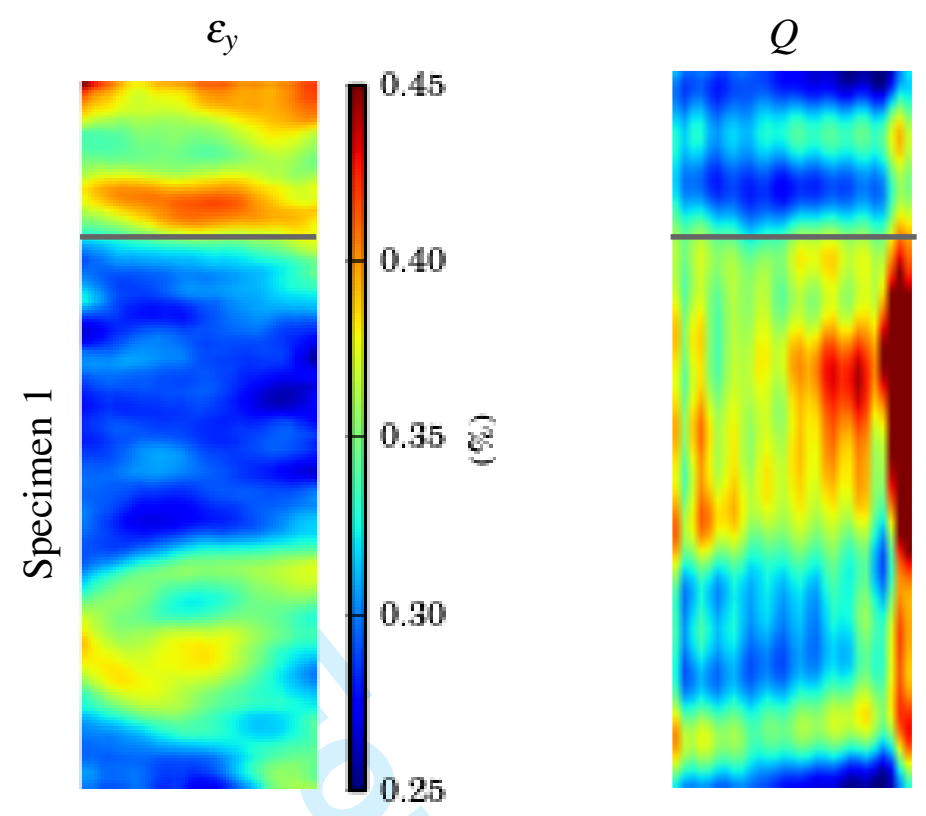

\section{Smoothed $Q$}
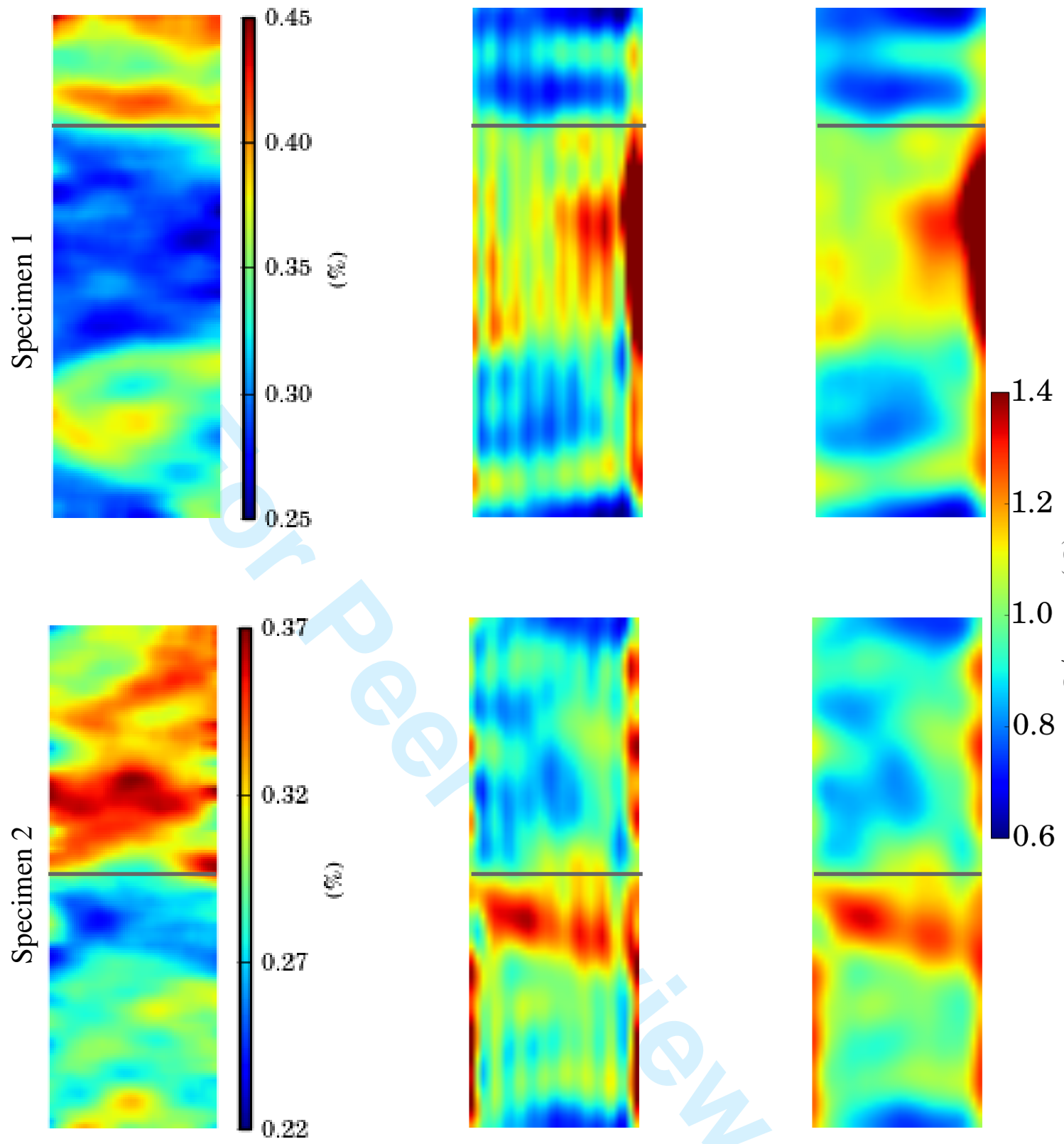

Figure 24: Strains and stiffnesses for each paperboard specimen. Rbf smoothing $=15$. Horizontal gray lines represent median vertical failure location. 